"This is an Accepted Manuscript of an article published by Macromol. Theory and Simul., mats.201700341, accepted in June 2017; vol 26, no 5, 1700041 (2017)."

\title{
Visualization of bivariate sequence length-chain length distribution in free radical copolymerization
}

Mohammad Reza Saeb ${ }^{1}$, Yousef Mohammadi ${ }^{2}$, Hadi Rastin ${ }^{3}$, Tayebeh Sadat Kermaniyan ${ }^{4}$, and Alexander Penlidis ${ }^{5 *}$

1. Department of Resin and Additives, Institute for Color Science and Technology, P.O. Box 16765654, Tehran, Iran

2. Petrochemical Research and Technology Company (NPC-rt), National Petrochemical Company (NPC), P.O. Box 14358-84711, Tehran, Iran

3. School of Chemical Engineering, College of Engineering, University of Tehran, P.O. Box 111554563, Tehran, Iran

4. Department of Polymer Engineering and Color Technology, Amirkabir University of Technology, Tehran, Iran

5. Department of Chemical Engineering, Institute for Polymer Research (IPR), University of Waterloo, Waterloo, Ontario, N2L 3G1 Canada

*To whom correspondence should be addressed:

Dr. Yousef Mohammadi: mohammadi@npc-rt.ir

Prof. Alexander Penlidis: penlidis@uwaterloo.ca 


\section{GRAPHICAL ABSTRACT}

Bivariate dynamic sequence length-conversion and end-of-batch sequence length-chain length distributions of free radical copolymerization under various monomer reactivity ratios and feed compositions are visualized by Kinetic Monte Carlo simulation.
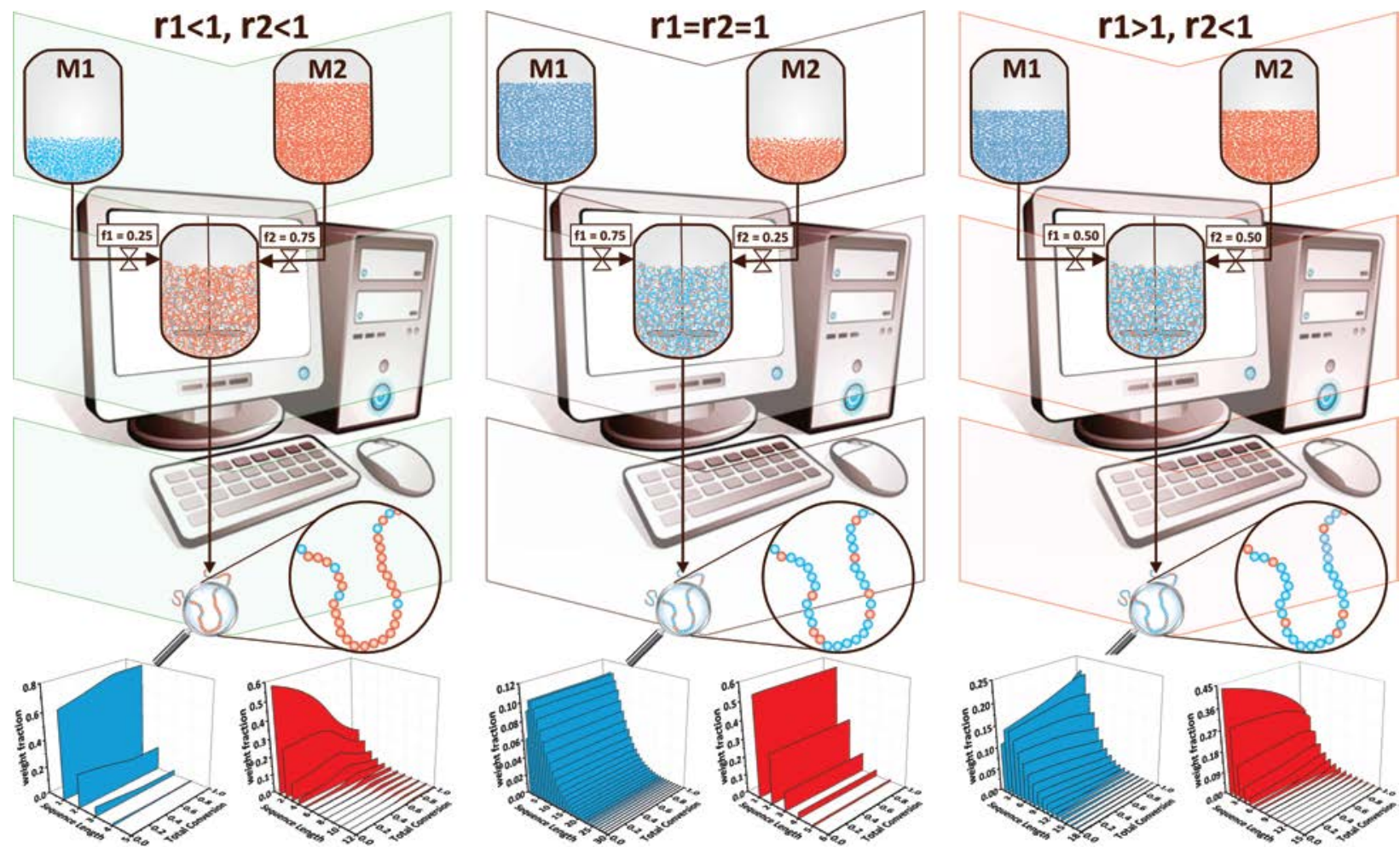


\section{ABSTRACT}

Copolymer properties and processability depend on copolymer microstructure, i.e., copolymer composition and monomer unit arrangements along copolymer chains. To predict ultimate properties of copolymers, one needs complete information on the length and position of sequences of each monomer type, $M 1$ and $M 2$, in every polymer chain in the system. A versatile Kinetic Monte Carlo (KMC) code has been developed and applied for the simulation of typical free radical copolymerizations. The code allows explicit monitoring of every growing chain during the course and at the end of polymerization, and can account for comonomer systems of any arbitrary reactivity ratios ( $r_{1}$ and $r_{2}$ ) over the full range of monomer composition. Meanwhile, it eliminates the need for solving arrays of differential equations arising from deterministic modeling approaches. Since the code virtually synthesizes billions of copolymer molecules and keeps in storage information on each and every copolymer chain in the system, it allows for detailed statistical analysis. The simulator visualizes the bivariate sequence length-chain length (SL-CL) distribution for typical copolymerization systems and examples with: $r_{1}<1 \& r_{2}<1 ; r_{1}>1$ \&

$r_{2}<1 ;\left(r_{1} \times r_{2}\right)=1$; and $r_{1}=r_{2}=1$, and is also applied successfully to an experimental scenario described in the literature.

Keywords: Monte Carlo simulation; Copolymerization; Chain length-Sequence length distribution; Molecular architecture; Tailor-made copolymers 


\section{INTRODUCTION}

A common characteristic of free radical copolymerization is composition drift [1-3]. In general, the nature of comonomers (as reflected by their different reactivity ratios), the initial feed composition of the polymerizing mixture, and the employed reactor configuration and operating conditions, all influence the evolution of microstructure of growing chains, and lead to the formation of diverse polymer products having different properties [4].

The microstructure of a copolymer can be identified by the number and position of monomers in the chains [5]. From this standpoint, the level of success in controlling ultimate product properties is largely dependent on the extent to which copolymer composition and monomer sequences are appropriately manipulated. The first step toward this objective is to have powerful mathematical tools capable of explicit 'monitoring' of macromolecular features of copolymer chains at any given interval of the copolymerization [6].

The need for tracking sequence length distribution (SLD), chain length distribution (CLD), and hence the bivariate sequence length-chain length (SL-CL) distribution of copolymers has recently been discussed by Kryven and ledema [7]. They have succeeded, by solving arrays of differential equations, in computing the length and sequence of styrene-acrylonitrile copolymers via numerical integration of two-dimensional population balance equations, and provided useful information about the bivariate SL-CL distribution. Their model permits fractionating copolymer chains in the system and enumerating sequences of each monomer type, from which one can calculate the mole fraction of different sequences. The authors obtained detailed patterns on chain composition using the developed detailed deterministic model. It must be kept in mind, however, that a population balance permits, at best, the calculation of frequencies of sequences of different lengths for different chain length fractions. It can address neither the position of sequences nor their inter- and intra-chain distribution. To make this clearer, suppose that we have two samples obtained from free radical copolymerization of monomer $1(M 1)$ and monomer 2 (M2) under the same operating conditions (see Scheme 1). Suppose also that the chain length 
(CL) of copolymer molecules takes on only the two values of 10 and 20 , but with different frequencies. At the same time, the number of double and triple sequences (i.e., diads and triads) of $M 2$, sequence length $S L=2$ and $S L=3$, take on the values of 3 and 8 in the two batches, respectively (as per Scheme 1).

Batch (I)

0000000000

0000000000

0000000000

0000000000

0000000000

0000000000

00000000000000000000

000000000000000000000

000000000000000000000

000000000000000000000

\section{0,0000000000000000000}

- Monomer M1
Batch (II)

0000000000

0000000000

0000000000

0000000000

00000000000000000000

000000000000000000000

0000000000000000000000

00000000000000000000

000000000000000000000

00000000000000000000

- Monomer M2

Scheme 1. Schematic of two batches obtained from a particular free radical copolymerization with only double and triple sequences of monomer $M 2$ on chains having constant length of 10 or 20; but with different placement/distribution of $M 1$ and $M 2$ sequences 
The simple illustration of Scheme 1 represents the concept of SL and CL and demonstrates the inability of deterministic population balances to account for the prediction of SL position as well as SL inter-and intra-chain distribution. Suppose the objective is to calculate the mole and weight fraction of triple $M 2$ sequences located on the chains with $C L=20$, i.e., the blocks specified with dotted rectangles in Scheme 1. The desired mole fraction, which takes on equal values for both samples, can be defined and calculated via equation (1), as the ratio of the number of triple $M 2$ sequences in copolymer chains with $\mathrm{CL}$ of 20 to the total number of $M 2$-type sequences, including double and triple sequences, as follows:

$S L-C L_{n}(3,20)=\frac{5}{8+3}=0.454$

Likewise, and assuming that $M W_{M 2}$ is the molecular weight of the $M 2$ unit, the weight fraction of triple $M 2$ sequences of chains having $C L$ of 20 can be calculated via equation (2):

$S L-C L_{w}(3,20)=\frac{5 \times\left(3 M W_{M 2}\right)}{8 \times\left(3 M W_{M 2}\right)+3 \times\left(2 M W_{M 2}\right)}=0.50$

Although the triple $M 2$ sequences in the two batches have the same mole and weight fraction, the properties of the two samples should be completely different considering that homopolymers of $M 1$ also exist in the system and triple $M 2$ sequences have different positions from one copolymer chain to the other. In a real case, we have billions of chains each having its particular molecular fingerprint $[8,9]$. In such a case, the presence of M1-type homopolymers together with random localization of $M 2$ sequences with different lengths (single, double, triple, quadruple, ...) at the end and/or at some location in the middle of the copolymer chains might cause significant differences in processability as well as in thermal, rheological, mechanical and biological properties of the resulting copolymers (e.g., see $[10,11])$. Thus, a more detailed tool is required for reliable simulation of microstructural copolymerization characteristics with the ability to explicitly track all copolymer chains in the system. 
Kinetic Monte Carlo (KMC) simulations give complete information about the microstructural chain evolution. This stochastic approach allows for detailed simulation of copolymerization chain characteristics [12-15]. With KMC, it is possible to assign an identification card to any single chain in the polymerizing mixture, which subsequently allows for monitoring, fractionating, visualizing, and screening of copolymer chains [16, 17].

In a previous work, we developed a KMC algorithm to capture the bivariate distribution of chain length and copolymer composition [18]. The algorithm affords the detection of any single growing chain in the polymerization system and can precisely categorize chains according to the number, weight, and position of comonomer units randomly positioned in copolymer chains. By giving the mother KMC simulator the ability of monitoring and reporting the length and position of sequences of each type existing in any individual copolymer chain, the present work aims to pattern the SL-CL distribution of copolymer chains stochastically via simulating a typical free radical copolymerization (initiation, propagation, chain transfer, and termination).

The present KMC simulator provides a detailed 'copy' of all copolymer chains synthesized during the course of polymerization. It can track the length and position of comonomer sequences, as a function of feed composition and reactivity ratios $\left(r_{1}\right.$ and $\left.r_{2}\right)$ for different ratio combinations $\left(r_{1}>1\right.$ $\& r_{2}<1 ; r_{1}<1 \& r_{2}<1 ;\left(r_{1} \times r_{2}\right)=1$ and $\left.r_{1}=r_{2}=1\right)$. The code is finally applied to visualize the SL-CL distribution of an experimental case previously studied in the literature. The present work helps engineers and scientists to acquire a comprehensive view of microstructural developments during the course of copolymerization.

\section{KINETIC MONTE CARLO SIMULATION}

Arrangement of monomer units along growing copolymer chains during polymerization depends on polymerization conditions and the values of the monomer reactivity ratios. In general, a typical free radical copolymerization with monomers $M 1$ and $M 2$ can be adequately described by the steps of Scheme 2 (e.g., see [19]). The quantity and the types of growing chains, i.e., homopolymer or copolymer chains, together with random localization of single or block units of M2 monomer, are the result of dissimilar tendency of monomers to add to the growing radicals. This indeed makes the detection of sequences of different types complex. Thus, the code is 
required to be computationally time-effective to be able to probe and fractionate all chains in the system on account of length and position of sequences of $M 1$ or $M 2$ type. This could be a serious challenge if one wanted to make the code versatile for quantification of any type of chains.

\begin{tabular}{|c|c|c|}
\hline (1) & Initiator dissociation & $I \stackrel{k_{d}}{\longrightarrow} 2 f P R^{\bullet}$ \\
\hline$(2,3)$ & Initiation & $P R^{\bullet}+M_{j} \stackrel{k_{i}}{\longrightarrow} R_{1, j}^{\bullet}$ \\
\hline$(4-7)$ & Propagation & $R_{n, i}^{\bullet}+M_{j} \stackrel{k_{p, i j}}{\longrightarrow} R_{n+1, j}^{\bullet}$ \\
\hline (8) & Termination by combination & $R_{n, i}^{\bullet}+R_{m, j}^{\bullet}$ \\
\hline (9) & Termination by disproportionation & $R_{n, i}^{\bullet}+R_{m, j}^{\bullet} \stackrel{k_{t d}}{\longrightarrow} P_{n}+P_{m}$ \\
\hline \multicolumn{3}{|c|}{$i, j: 1$ or 2} \\
\hline \multicolumn{3}{|c|}{$P R^{\bullet}$ : Primary radical } \\
\hline \multicolumn{3}{|c|}{$f:$ Initiator efficiency } \\
\hline \multicolumn{3}{|c|}{$I, M_{j}$ : Initiator molecule and monomer/comonomer, respectively } \\
\hline \multicolumn{3}{|c|}{$R_{n, i}^{\bullet}$ : Macroradical with $n$ repeating units ending in $i$-type monomer } \\
\hline \multicolumn{3}{|c|}{$P_{n}:$ Copolymer chain with $n$ repeating units } \\
\hline
\end{tabular}

Scheme 2. Reaction scheme applied in KMC simulation of free radical copolymerization of typical $M 1$ and $M 2$ monomers for cases having different reactivity ratios

We specified the kinetic parameters involved based on typical values from the literature and applied them to the simulation of a typical free radical copolymerization (Table 1) [19]. For all cases simulated in this work, the final conversion was set to $100 \%$.

Table 1. Parameters used in KMC simulation of typical free radical copolymerization of $M 1$ and M2 monomers for cases having different reactivity ratios

\begin{tabular}{|c|c|c|}
\hline Parameter & Value & Units \\
\hline Initiator dissociation rate constant $\left(\boldsymbol{k}_{\boldsymbol{d}}\right)$ & $10^{-3}$ & $\sec ^{-1}$ \\
\hline Initiator efficiency $(\boldsymbol{f})$ & 0.50 & - \\
\hline Chain initiation rate constant $\left(\boldsymbol{k}_{\boldsymbol{i}}\right)$ & $10^{4}$ & lit $\mathrm{mol}^{-1} \mathrm{sec}^{-1}$ \\
\hline Homo-propagation rate constant $\left(\mathrm{A}, \boldsymbol{k}_{p, 11}\right)$ & $10^{4}$ & lit $\mathrm{mol}^{-1} \mathrm{sec}^{-1}$ \\
\hline Homo-propagation rate constant $\left(\mathrm{B}, \boldsymbol{k}_{p, \mathbf{2 2}}\right)$ & $10^{4}$ & lit $\mathrm{mol}^{-1} \mathrm{sec}^{-1}$ \\
\hline Termination by combination rate constant $\left(\boldsymbol{k}_{t c}\right)$ & $10^{8}$ & lit $\mathrm{mol}^{-1} \mathrm{sec}^{-1}$ \\
\hline
\end{tabular}


Termination by disproportionation rate constant $\left(\boldsymbol{k}_{\boldsymbol{t} d}\right) \quad 10^{8} \quad$ lit $\mathrm{mol}^{-1} \mathrm{sec}^{-1}$ Initial monomer-to-initiator molar ratio $100-$

In this work, the free radical copolymerization of $M 1$ and $M 2$ monomers was simulated at three different initial feed compositions (i.e., 25,50 , and 75 mole \% of $M 1$ monomer) for cases with different reactivity ratios: $r_{1}<1 \& r_{2}<1 ; r_{1}>1 \& r_{2}<1 ;\left(r_{1} \times r_{2}\right)=1$; and $r_{1}=r_{2}=1$. The different distribution varieties of chains and sequences in the system are due to the reactivity ratios. The different cases simulated were initially used for visualization and comparison of SL-CL distribution patterns as functions of initial feed composition, and later to confirm the reliability of subsequent simulation results.

To develop a versatile KMC simulator capable of quantifying 3D SL-CL distributions, Gillespie's algorithm was utilized to virtually synthesize linear binary copolymer chains via free radical copolymerization and precisely determine the microstructure of the generated macromolecules. Obviously, the type and initial concentration of monomers significantly influence the number, length, and position of both sequences of $M 1$ and $M 2$ in the produced copolymer chains. Basically, in all copolymerization processes, the architectural and topological features of the produced macromolecules are mainly influenced by the homo- and cross-propagation reaction channels. In order to select a macroradical for propagation, a selection probability was assigned to each growing chain existing in the simulation volume. A random number, $r$, was generated and the $m^{\text {th }}$ growing chain of type $h$ (terminal monomer unit) was selected for propagation provided the following criterion was satisfied:

$$
\sum_{j=1}^{m-1} p_{j, h} \leq r \leq \sum_{j=1}^{m} p_{j, h}, \quad p_{j, h}=\frac{1}{R_{h}}
$$

In this criterion, $p_{j, h}$ is the selection probability of the $j^{\text {th }}$ growing chain of type $h$ and $R_{h}$ is the total number of macroradicals of type $h$ in the simulation volume. Typically, in linear binary free radical copolymerization, two distinct types of macroradicals, i.e., radicals ending in $M 1$ or $M 2$ units, can be recognized in the medium. To propagate the selected growing chain at the given time interval, the selection probabilities of $M 1$ and $M 2$ were determined based on the instantaneous 
propagation reaction rates relative to the total polymerization rate. In this way, the incorporation probability of monomer $M 1$ and comonomer $M 2$ to the growing chains was precisely determined. Considering the classical statistical copolymerization equations, these probabilities are related to the reactivity ratios and concentrations (or mole fractions) of unreacted monomer/comonomer at that specific moment.

The selection mechanism of radical chains to participate in termination channels is similar to the aforementioned mechanism employed to simulate the propagation channel. These termination reactions have a prominent role in controlling the average molecular weight and molecular weight distribution.

The number of initial $M 1$ molecules was chosen as the basis to define and construct the simulation volume. To do this, a total of $10^{12} \mathrm{M} 1$ monomer units was used as the initial input value to the computer code and the number of other reactants was computed based upon the copolymerization recipe, i.e. initial feed composition and initial monomer-to-initiator molar ratio. In previous work, we described the procedure by which placement of $M 2$ comonomer was monitored [18]. The computational algorithm developed in this work addresses the need for monitoring the length and position of sequences of both monomer types, as demonstrated in Scheme 3. Each copolymer chain in the polymerization system contains a number of $M 1$ and $M 2$ sequences with fixed lengths (i.e., static sequences). The terminal sequence on the active-side of a growing chain is 'alive' (i.e., a dynamic sequence) and its length varies with the course of copolymerization before experiencing a cross-propagation or termination event. Hence, a welldesigned data storage structure should be designed to store all architectural information necessary to completely visualize a copolymer chain. To do this, a novel algorithm was proposed capable of storing all instantaneous characteristics of dynamic last sequences along with all cumulative information of static sequences on the same chain. This algorithm allowed the simulation of a statistically large sample size with a computationally cost-effective execution time. 


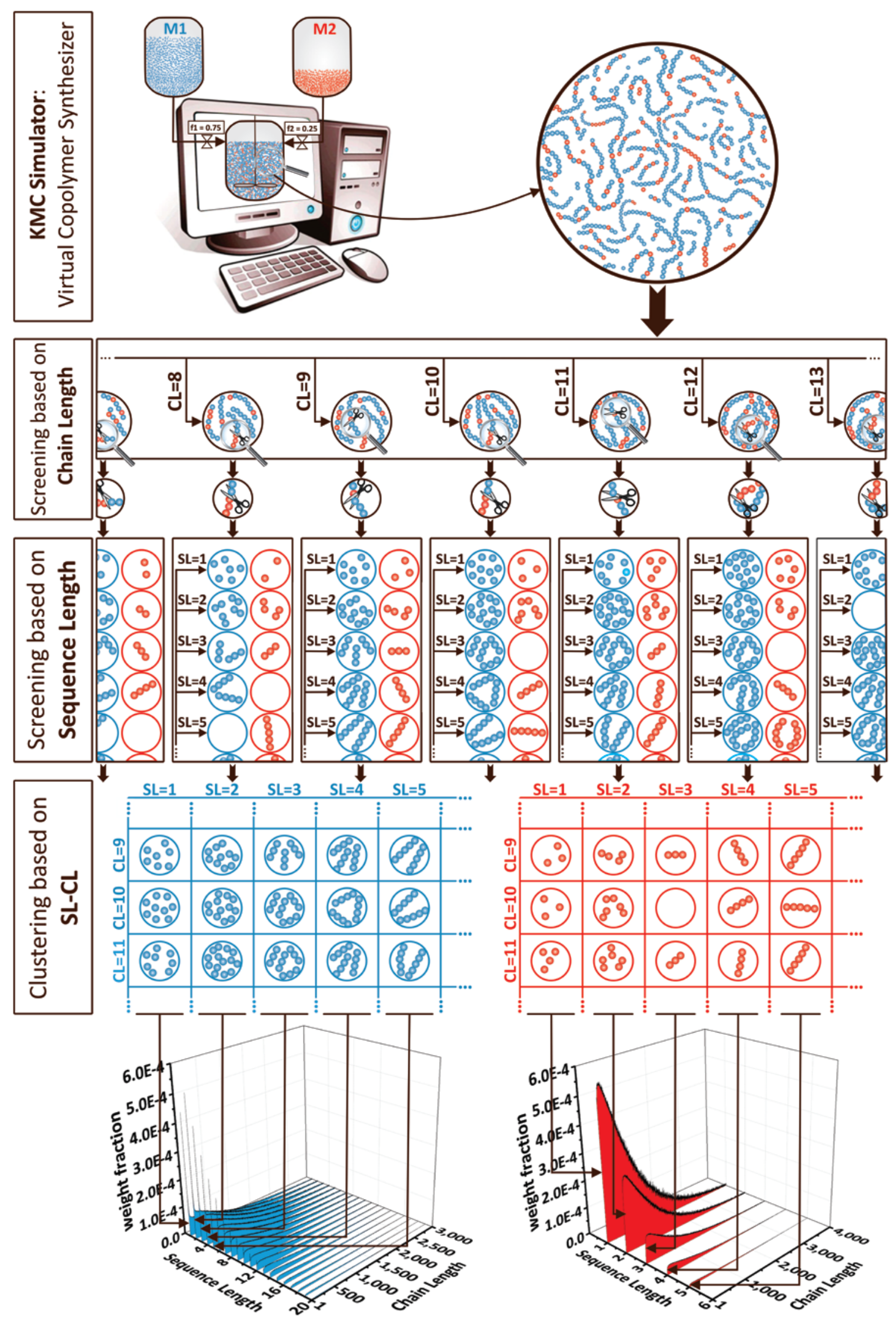


Scheme 3. Schematic representation of the algorithm applied to (a) synthesize copolymer chains through free radical copolymerization of typical $M 1$ and $M 2$ monomers and (b) visualize bivariate SL-conversion and SL-CL distributions

The computer program was written in Pascal programming language (Lazarus 1.2.4 IDE) and compiled into 64-bit executable code using FPC 2.6.2. A sub-routine based on the "Mother-of-all Pseudo-Random Number Generators" algorithm was employed to produce the required random numbers for the simulation [20]. The random number generation subroutine satisfied the tests of uniformity and serial correlation with high resolution. The cycle length of the random number generator was $3 \times 10^{47}$. Simulations were performed with a desktop computer with Intel Core i73770K (3.50 GHz), $32 \mathrm{~GB}$ of memory (2133 MHz), under Windows 7 Ultimate 64-bit operating system. The runtime approximately took between 5 and 7 hours.

\section{RESULTS AND DISCUSSION}

It is well-documented that random copolymerization exhibits minimum compositional drift among all types of reactions, when values of reactivity ratios are nearly equal to unity, $r_{1}=r_{2}=1$. In such a case, there is no preference for the propagating species $M 1^{*}$ and $M 2^{*}$ to capture monomers of the same or different type. On the other hand, when we have different reactivity ratios, i.e., $r_{1}>1 \& r_{2}<1$, or $r_{1}<1 \& r_{2}<1$, composition drift is expected. Such compositional drifts can be, more or less, intensified/moderated by feed composition and/or by the relative difference of the reactivity ratios. Comparison of different copolymerizations with various reactivity ratios and feed compositions would be helpful to visualize the evolution in microstructure of chains during the course (and at the end) of the batch. Typical copolymerizations were simulated in accord with reaction channels presented in Scheme 2 and kinetic parameters given in Table 1. Investigations on variation patterns on concentration of reactants and products (monomers, polymers, macroradicals, and initiator), consumption rate of monomers, the number- and weight-average degree of polymerization and polydispersity indices all give useful information about the general features of the polymerization in terms of time or conversion. Then, 3D plots on weigh fraction of chains in terms of conversion and SL, and 
bivariate SL-CL distribution of end-of-batch products are patterned and discussed. Finally, the aforementioned 3D plots are visualized for an experimental copolymerization accompanied by chain transfer to monomer.

\subsection{Simulation of Classical Copolymerization Kinetics}

\subsubsection{Case (I) with $r_{1}<1 \& r_{2}<1$}

In many cases, practitioners would like copolymerizations to run with the lowest possible compositional drift $[21,22]$. With $f_{1}$ and $F_{1}$, respectively, as the instantaneous mole fraction of $M 1$ in the feed and copolymer, the best condition would be $F_{1}=f_{1}=f_{1,0}$ over a broad range of conversion ( $f_{1,0}$ is the initial feed composition). Thus, the code has been developed in a manner to capture fluctuations in microstructure of growing chains for systems with specified reactivity ratios at different initial feed compositions, i.e., $\mathrm{f}_{1,0}=0.25,0.50$, and 0.75 .

Polymerization of monomers with $r_{1}<1 \& r_{2}<1$ leads to production of copolymers with regular alternating $M 1$ and $M 2$ units, because the propagating species $M 1^{*}$ and $M 2^{*}$ prefer to capture monomers of a different type. Thus, depending on the value of $f_{1,0}$, composition drift will take place mildly or considerably. The copolymerization products between acrylonitrile and acrylamide $\left(r_{1}=0.86 \& r_{2}=0.81\right) ;$ maleic anhydride and vinyl acetate $\left(r_{1}=0.0 \& r_{2}=0.019\right) ;$ and styrene and methyl methacrylate $\left(r_{1}=0.52 \& r_{2}=0.46\right)$ are examples of copolymers with such an alternating tendency (e.g., see [19]). We used $r_{1}=0.7$ and $r_{2}=0.1$ in the simulation of a typical copolymerization as representative of this type of macromolecules.

Figure 1 shows generic features of the product from such a typical copolymerization with $r_{1}=0.7$ and $r_{2}=0.1$ under different feed compositions. From Figure $1 \mathrm{~A}$ it can be observed that monomer $M 1$ has been consumed faster than monomer $M 2$ due to the greater value of its reactivity ratio. It can be also recognized that $M 1$ has remained for a longer period in the system as the feed composition increased from of 0.25 to 0.50 to 0.75 (Figure $1 \mathrm{~A}$ to Figure $1 \mathrm{C}$ ). 
(A)

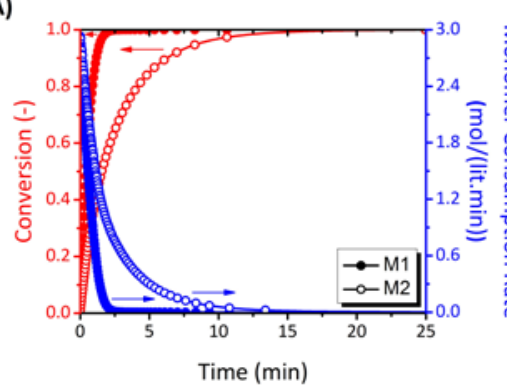

(B)

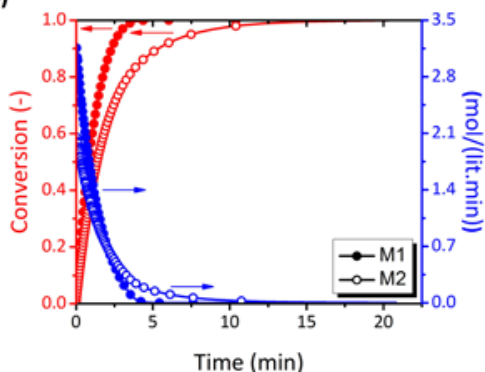

(C)

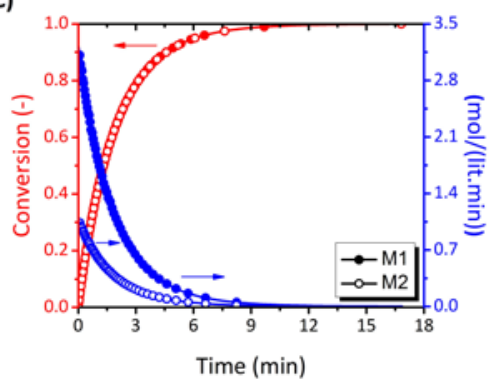

( $\left.A^{\prime}\right)$

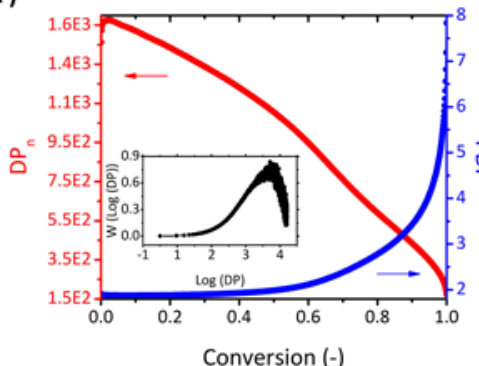

(B')

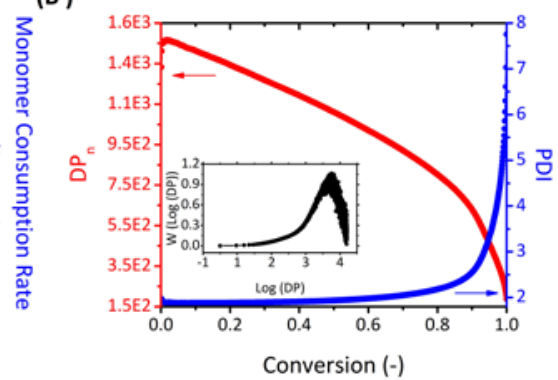

(C')

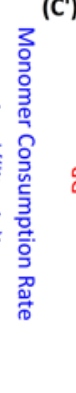

(A")

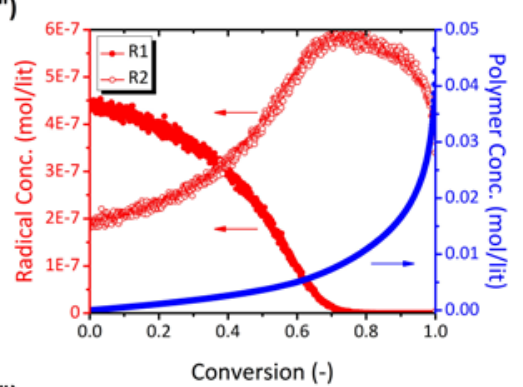

(B")

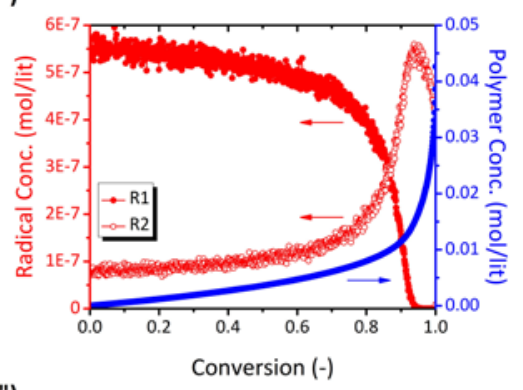

(C")

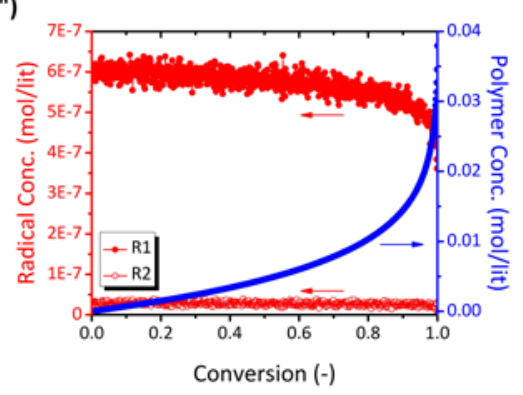

Figure 1. Profiles of monomer conversion and monomer consumption rates vs. time (A, B, C); variation of number-average degree of polymerization (DPn) and polydispersity index (PDI) of copolymer chains vs. conversion with inset of $\operatorname{CLD}\left(A^{\prime}, B^{\prime}, C^{\prime}\right)$; and variation of radical and polymer concentrations vs. conversion ( $\left.\mathrm{A}^{\prime \prime}, \mathrm{B}^{\prime \prime}, \mathrm{C}^{\prime \prime}\right) ; r_{1}=0.7$ and $r_{2}=0.1 ;\left(\mathrm{A}, \mathrm{A}^{\prime}, \mathrm{A}^{\prime \prime}\right)$ are for $f_{1}=0.25$; (B, $\left.\mathrm{B}^{\prime}, \mathrm{B}^{\prime \prime}\right)$ for $f_{1}=0.50$; and $\left(\mathrm{C}, \mathrm{C}^{\prime}, \mathrm{C}^{\prime \prime}\right)$ for $f_{1}=0.75$

It is interesting to emphasize that $M 1$ and $M 2$ coexist in the system under feed composition of 0.75 , as realized from the fact that conversion patterns in Figure $1 \mathrm{C}$ are superimposed on each other. This is due to the fact that a mixture of 75 mole percent of monomer $M 1$ in a system with $r_{1}=0.7$ and $r_{2}=0.1$ is the azeotropic composition. At this point, where $F_{1}=f_{1}$, no composition drift will take place.

The middle column in Figure 1 shows variation of the number-average degree of polymerization, $\mathrm{DP}_{\mathrm{n}}$, on the left-hand side vertical axis, and polydispersity index, PDI, on the right-hand side 
vertical axis, against conversion, while insets show the weight chain length distribution. From top to bottom, i.e., from Figure $1 A^{\prime}$ to Figure $1 C^{\prime}$, i.e., by increasing the amount of $M 1$ in the feed from 25 to 75 mole percent, the initial value of $D P_{n}$ decreases, due to the lower tendency of $M 1$ to homo-polymerize at higher concentrations. The third column of graphs shows variation of the concentration of the two types of radicals (designated by R1 and R2) and that of polymer against conversion. According to Figure $1 \mathrm{~A}^{\prime \prime}$, macroradicals of $M 1$ type, $\mathrm{R} 1$, are dominant in the system at the beginning of reaction, while faster consumption of $M 1$ upsets the balance toward $R 2$ at the later stages of reaction. This phenomenon was intensified toward higher conversions by increasing the feed composition from 0.25 to 0.50 (Figure 1A" to Figure 1B" ), but followed a completely different path in Figure $1 \mathrm{Cl}^{\prime}$. In the latter case, concentrations of R1 and R2 are almost constant over the full composition range, which is a special case like random copolymerization with reactivity ratios equal to unity. As a further evidence for the so-called azeotropic point, copolymer chains with less heterogeneity and lower PDI values, are produced [1].

Figure 2 shows variation of instantaneous copolymer composition $\left(F_{1}\right)$ and instantaneous mole fraction of diads (F11, F12, and F22) for the system with $r_{1}=0.7$ and $r_{2}=0.1$ at different feed compositions of $0.25,0.50$, and 0.75 vs. conversion. 
(A)

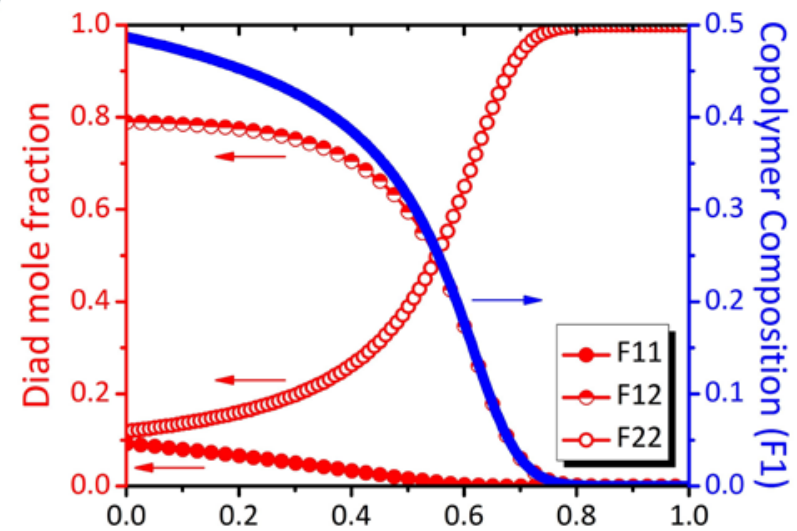

Conversion (-)

(B)

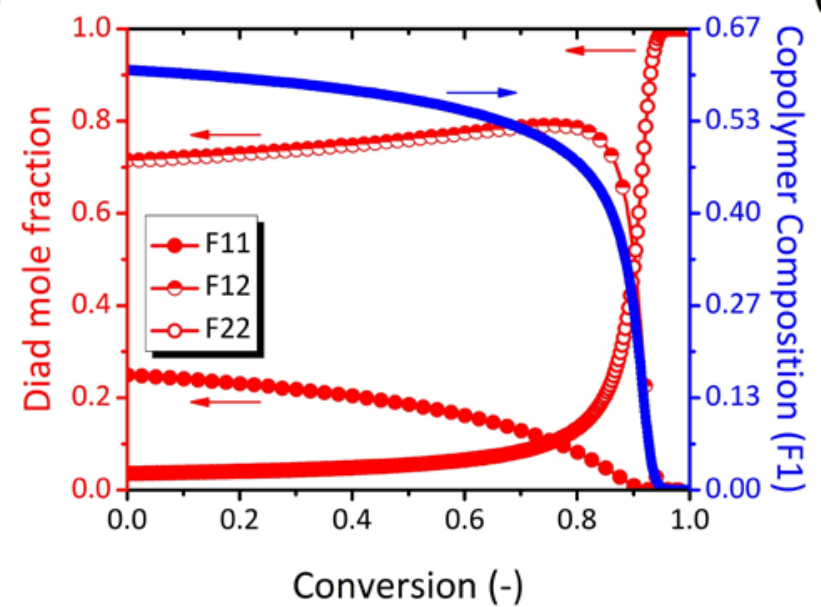

(C)

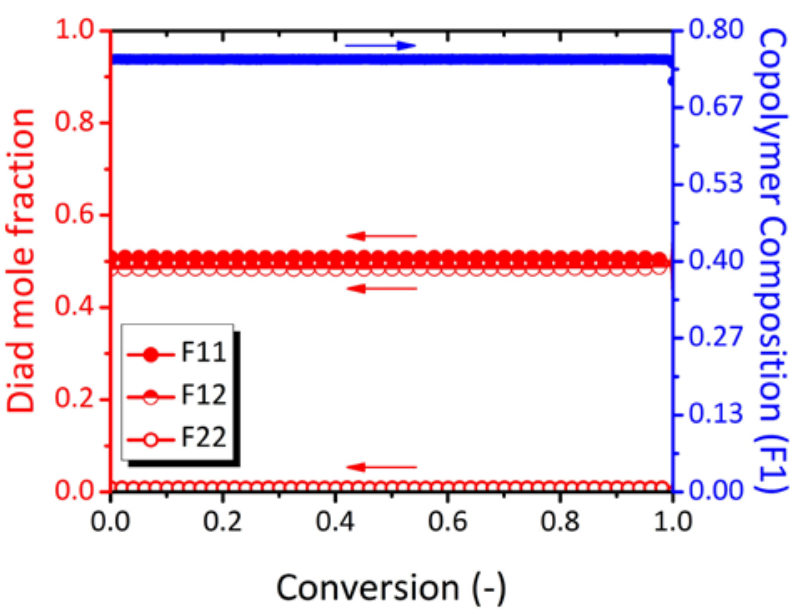

( $\left.A^{\prime}\right)$

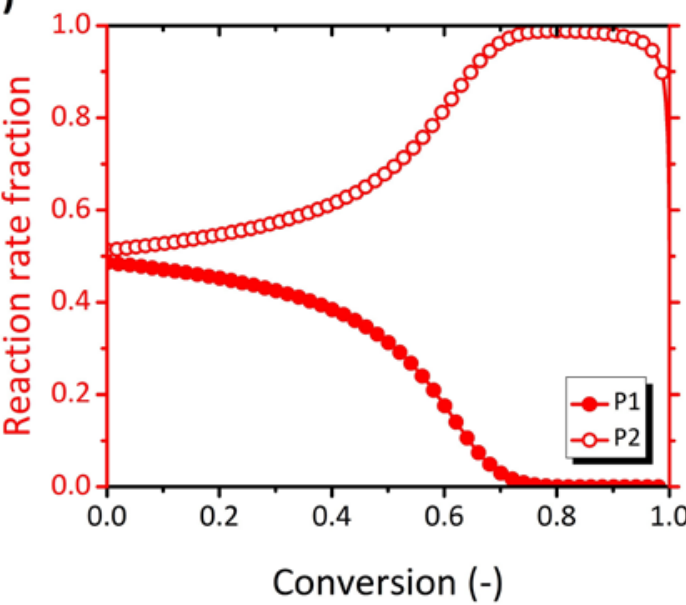

(B')

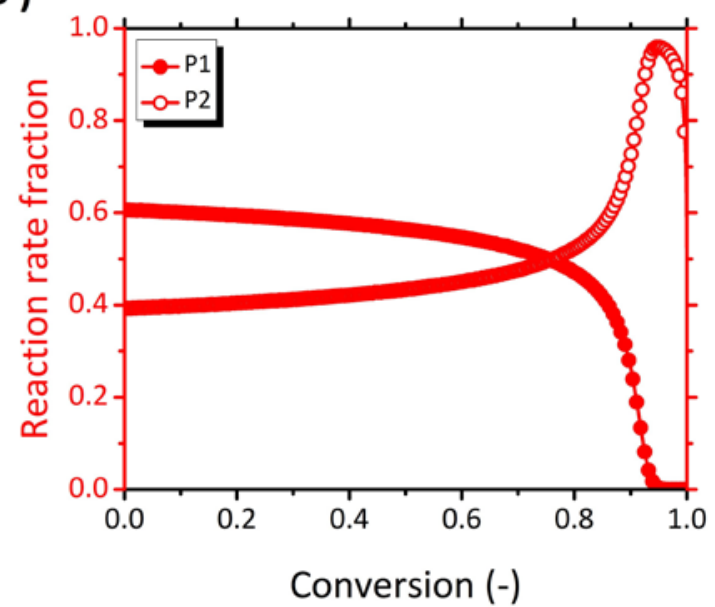

(C')

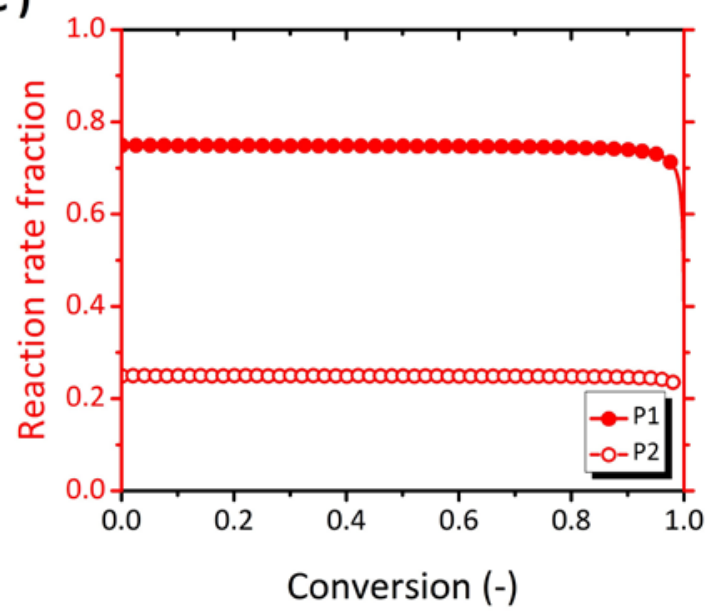

Figure 2. Profiles of instantaneous diad mole fractions (F11, F12, and F22) and instantaneous copolymer composition (F1) vs. conversion (A, B, C) and propagation reaction rate fraction (with 
$\mathrm{P} 1$ and $\mathrm{P} 2$ denoting propagating chains ending in $M 1$ and $M 2)$ vs. conversion $\left(A^{\prime}, B^{\prime}, C^{\prime}\right) ; r_{1}=0.7$ and $r_{2}=0.1 ;\left(\mathrm{A}, \mathrm{A}^{\prime}\right)$ are for $f_{1}=0.25 ;\left(\mathrm{B}, \mathrm{B}^{\prime}\right)$ for $f_{1}=0.50$; and $\left(\mathrm{C}, \mathrm{C}^{\prime \prime}\right)$ for $f_{1}=0.75$

In the case of 0.25 and 0.50 mole percent feed compositions (Figure 2A and Figure 2B), crosspropagation takes place more than homo-propagation at an early- to intermediate-stage of conversion in view of higher values of F12 than those of F11 and F22. In agreement with Figure $1 C^{\prime}$, the homo- and cross-propagation of $M 1$ are of the same order at azeotropic composition (Figure 2C), while homo-polymerization of $M 2$ is rather low (see very low mole fraction of the corresponding diads, F22). Moreover, diad mole fractions of all types take constant values at azeotropic condition.

The 3D plots of instantaneous variation of SL weight distribution vs. conversion and SL-CL distribution of end-of-batch product may shed additional light on the system. The dominance of cross-propagation at an early to intermediate stage in the case with 0.25 feed composition observed in Figure $2 \mathrm{~A}$ is now supported by the formation of chains with sequences having lengths not exceeding 4 (Figure $3 \mathrm{~A}$ ). When $M 1$ is consumed completely at an intermediate conversion, chains having longer sequences of $M 2$ are formed in the system (Figure 4A). Upon increasing the feed composition from 0.25 to 0.75 , moving from Figure $3 \mathrm{~A}$ to Figure $3 \mathrm{C}$, chains with longer sequences of $M 1$ type are formed. On the other hand, the lengths of sequences of $M 2$ illustrated in Figure 4B and Figure 4C follow an inverse trend. And more notably, the weight fraction patterns of sequences of different length, either $M 1$ or $M 2$, exhibit a rectangular shape at the azeotropic point (Figure 3C and Figure 4C). In support of this, $M 2$ single units in Figure $4 C$ have the highest weight fraction, which can be ascribed to cross-propagation with $M 1$. A more general case showing minimum compositional drift, irrespective of feed composition, is the random copolymerization of $M 1$ and $M 2$ with $r_{1}=1$ and $r_{2}=1$ (see Figure 11 and Figure 12 in the Appendix). Polymerization of ethylene and vinyl acetate $\left(r_{1}=0.97 \& r_{2}=1.02\right)$ is an example of such random copolymerization. In such a situation, the two monomers show equal reactivities toward the propagating species of both types; hence, homo- and cross-propagation coincide with random placement of monomers along the copolymer chains. The characteristic of this behavior is that 
the copolymer composition at any time during polymerization is the same with the one in the feed (e.g., see [19]).

Analysis of the SL-CL distribution of the end-of-batch product offers additional clarification of the behavior. The presence of long rods corresponding to very short chains in the system in Figure $3 C^{\prime}$, which is more obviously seen in the case of double and triple sequences, is a signature of homo-polymerization of $M 1$ at the azeotropic point. According to Figure $3 A^{\prime}$, single $M 1$ units are the main type of sequence in copolymer chains, whereas sequences of longer length can hardly be found. The rods representing homopolymers of $M 2$ type appear in the cases of 0.25 and 0.50 mole percent feed compositions in the SL-CL distribution patterns (Figure $4 A^{\prime}$ to Figure $4 C^{\prime}$ ), due to the accumulation of monomer $M 2$ at the final stages of these copolymerizations. 
(A)

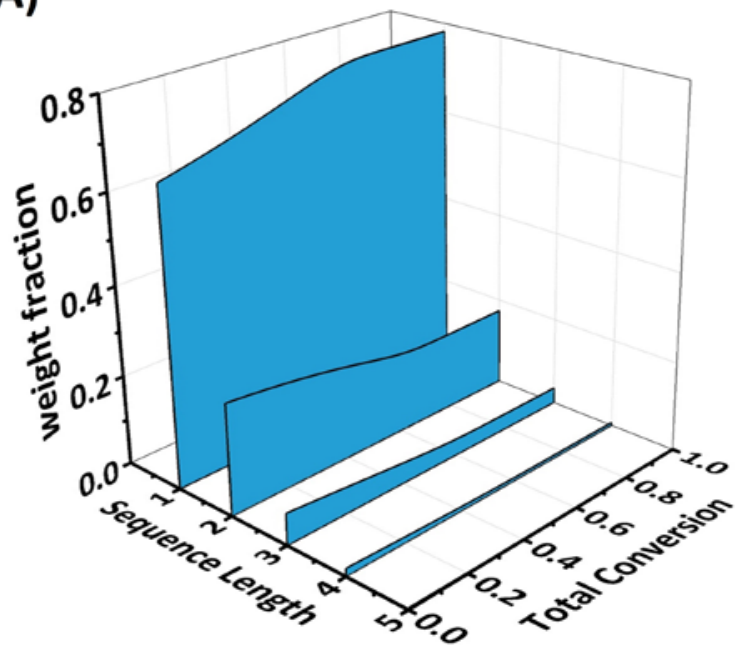

(B)

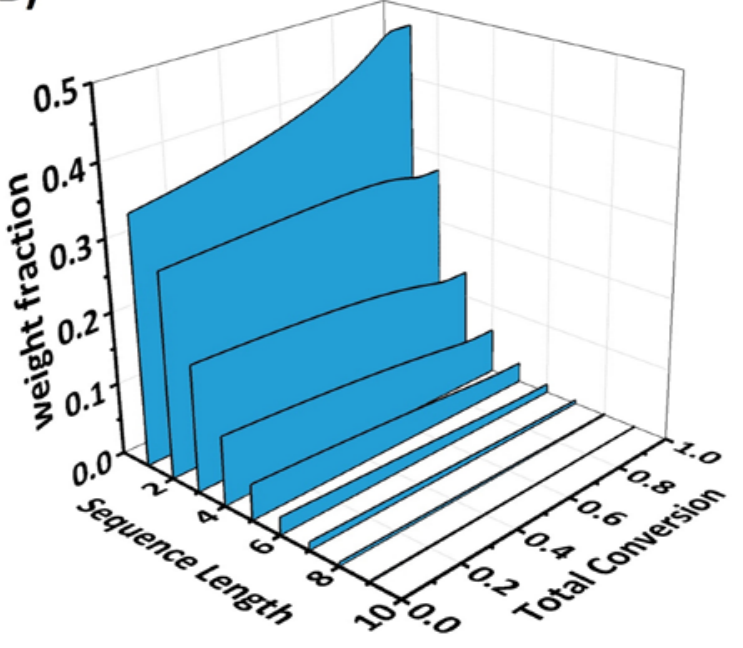

(C)

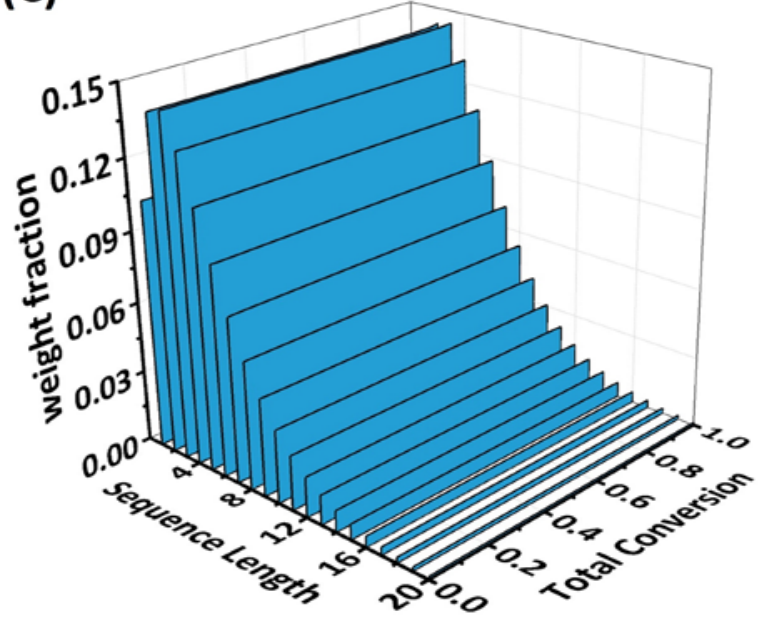

(A')

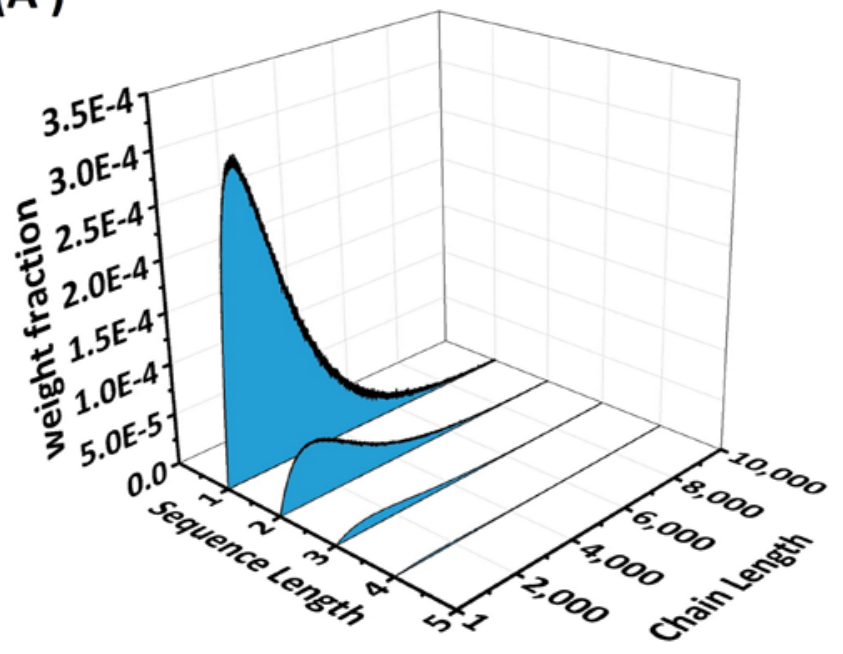

(B')

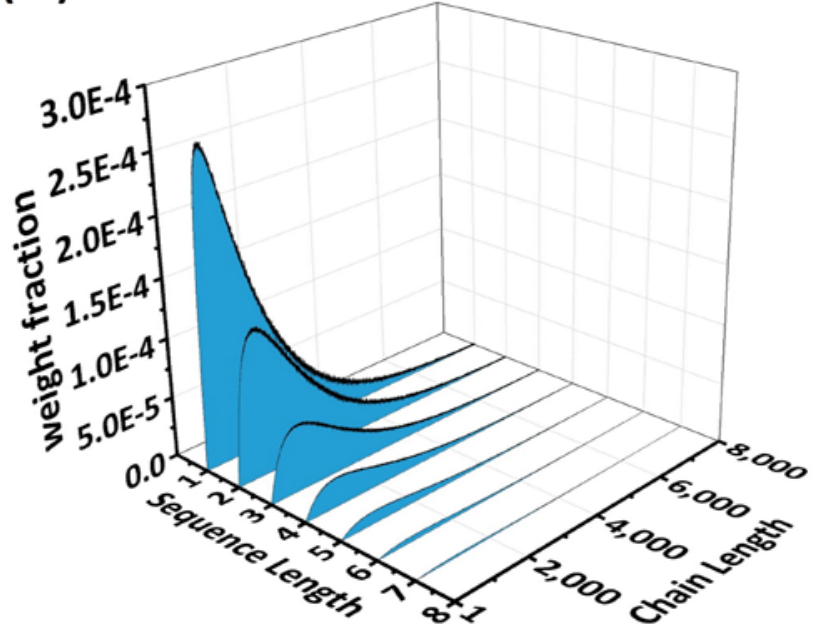

(C')

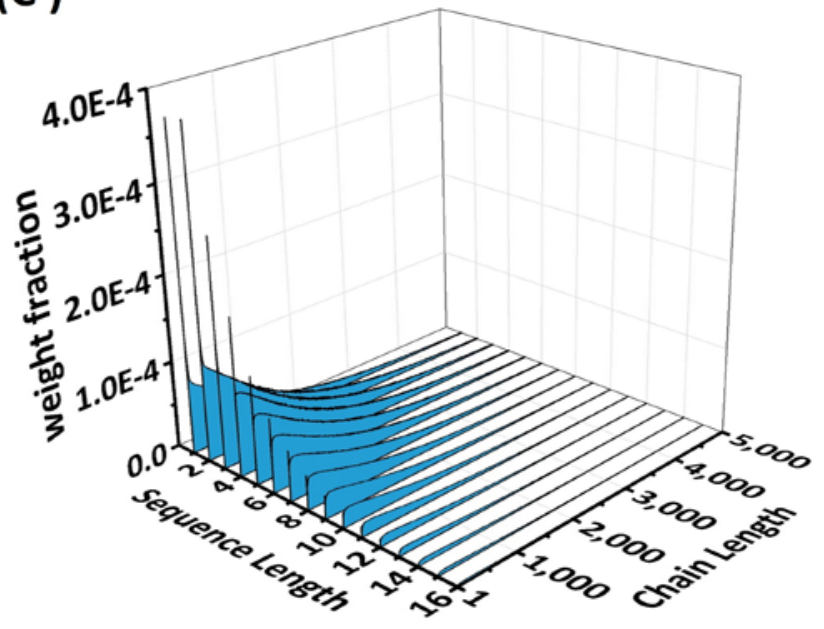


Figure 3. Profiles of evolution of weight fraction of sequence length distribution of $M 1$ under feed composition of 0.25 (A); 0.50 (B); and 0.75 (C) against conversion; and SL-CL weight distribution of $M 1$ for feed composition of $0.25\left(A^{\prime}\right) ; 0.50\left(B^{\prime}\right)$; and $0.75\left(C^{\prime}\right) ; r_{1}=0.7$ and $r_{2}=0.1$ 
(A)

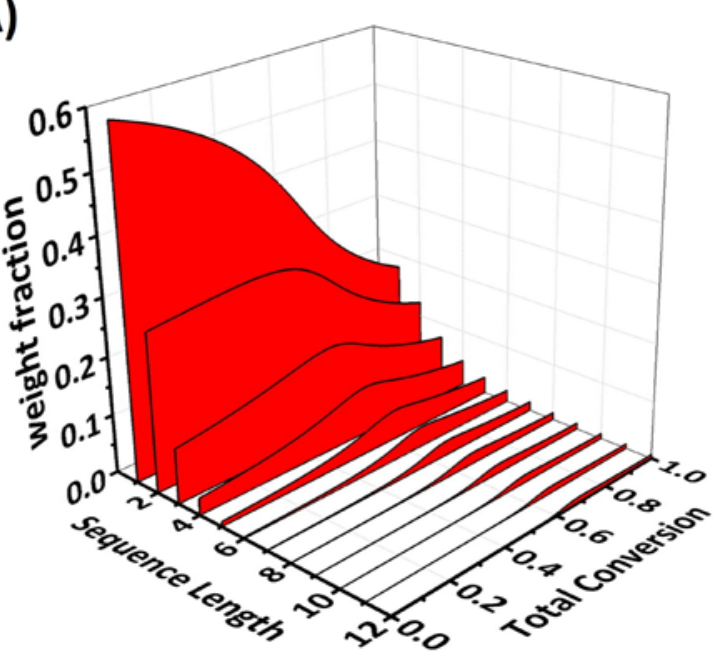

(B)

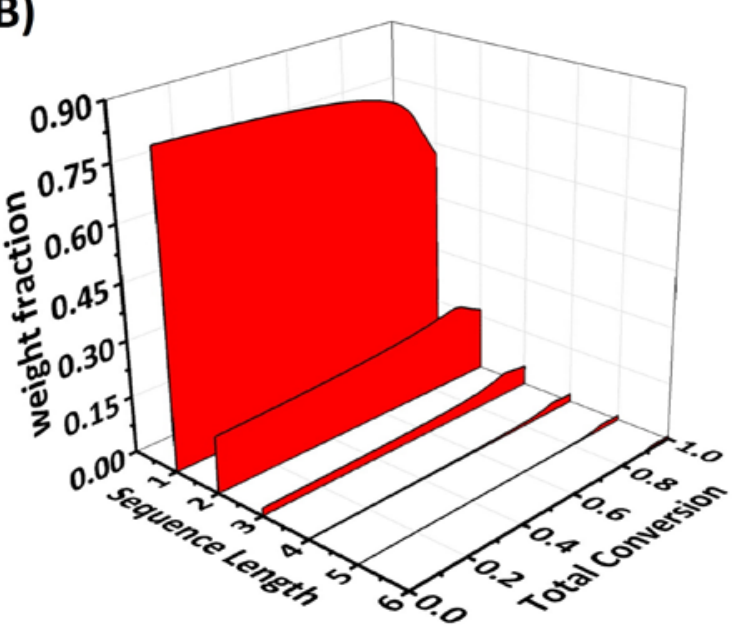

(C)

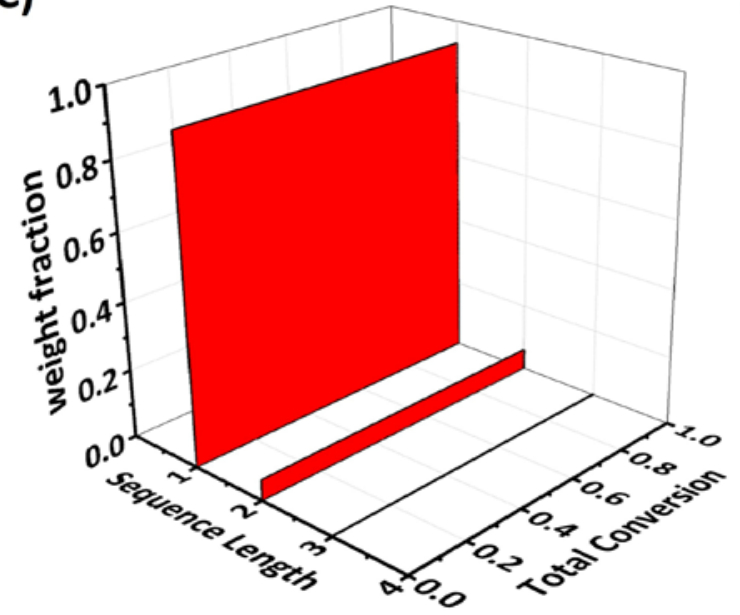

(A')

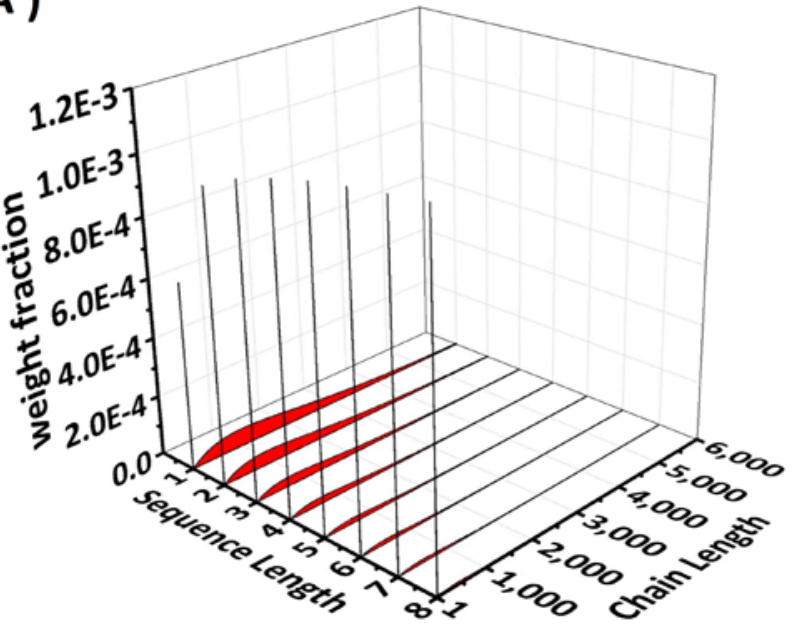

(B')

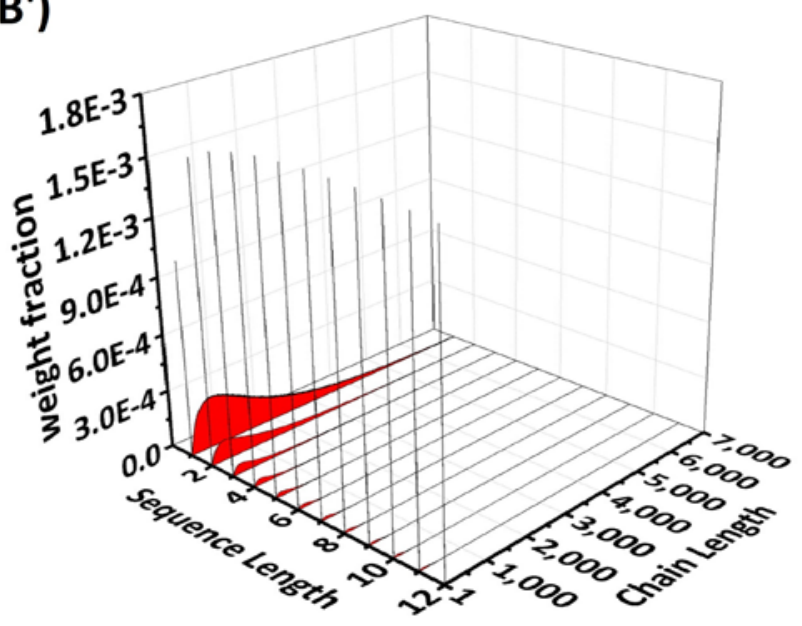

(C')

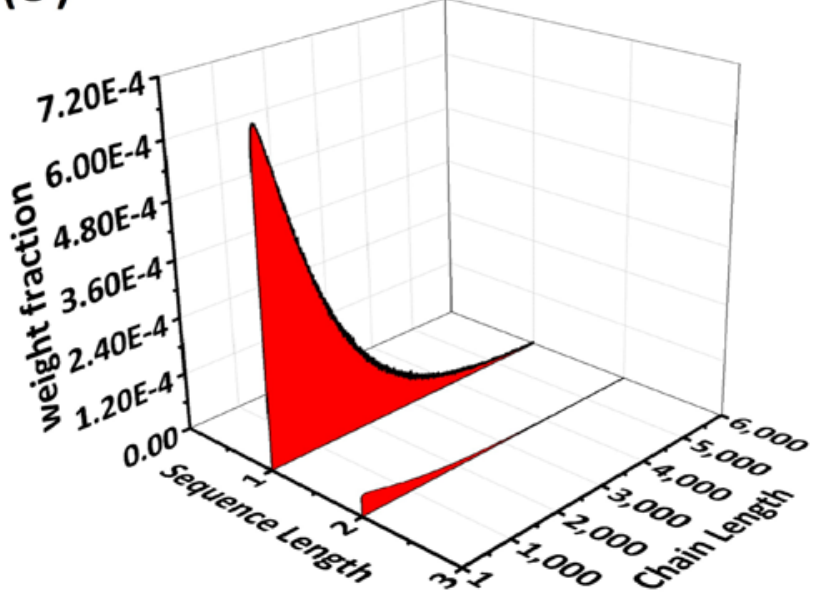


Figure 4. Profiles of evolution of weight fraction of sequence length distribution of $M 2$ under feed composition of 0.25 (A); 0.50 (B); and 0.75 (C) against conversion; and SL-CL weight distribution of $M 2$ for feed composition of $0.25\left(\mathrm{~A}^{\prime}\right) ; 0.50\left(\mathrm{~B}^{\prime}\right)$; and $0.75\left(\mathrm{C}^{\prime}\right) ; r_{1}=0.7$ and $r_{2}=0.1$

\subsubsection{Case (II) with $r_{1}>1 \& r_{2}<1$}

Another copolymerization with considerable compositional drift would be the case where we have monomers with $r_{1}>1 \& r_{2}<1$. In this case, monomer $M 1$ has a higher reactivity than $M 2$ toward the propagating species $\mathrm{M1}^{*}$ and $\mathrm{M} 2^{*}$; hence, the copolymer is expected to have a larger fraction of $\mathrm{M} 1$ in the macromolecular chains. Examples of monomer pairs with reactivity ratios of this category are acrylic acid and vinyl acetate $\left(r_{1}=8.7 \& r_{2}=0.21\right) ; 1$,3-butadiene and styrene $\left(r_{1}=1.4 \& r_{2}=0.58\right) ;$ styrene and vinyl chloride $\left(r_{1}=90 \& r_{2}=0.01\right)$; and vinylidene chloride and vinyl acetate $\left(r_{1}=4.7 \& r_{2}=0.03\right)$ [19]. In this study, a typical system with $r_{1}=5.0$ and $r_{2}=0.1$ is selected to be simulated as representative of the category. Since the value of $r_{2}$ is the same with the previous case, it is possible to compare compositional drift of the two cases on account of the different tendency of $M 1$ to homo- or cross-propagate.

Figure 5 shows typical profiles of generic features for the system with $r_{1}=5.0$ and $r_{2}=0.1$ at different feed compositions. Similar to the previous case, Figure $5 A$ shows that $M 1$ has been consumed more quickly than $M 2$ due to its higher reactivity ratio. Comparison of trends in Figure $5 A^{\prime}$ and Figure $5 A^{\prime \prime}$ shows that $D_{n}$ takes an almost linear downward trend from 470 to 360 over the conversion range $[0,0.50]$, and then experiences a plateau between conversions 0.50 to 0.70 , until the concentration of $M 2$ macroradicals overtakes that of $M 1$, followed by a sudden drop to 175 at the end of the polymerization. This 'shoulder' corresponds to an intermediate stage and will be revisited later. Figure $5 A^{\prime \prime}$ demonstrates that macroradicals of $\mathrm{R} 1$ type are abundant in the system in the beginning, while faster consumption of $M 1$ tips the balance toward R2 at the later stages of polymerization. Moreover, by increasing the feed composition from 0.25 to 0.75 (Figure $5 A^{\prime \prime}$ to Figure $\left.5 C^{\prime \prime}\right)$, the $R 1$ macroradicals play the major role. 
(A)

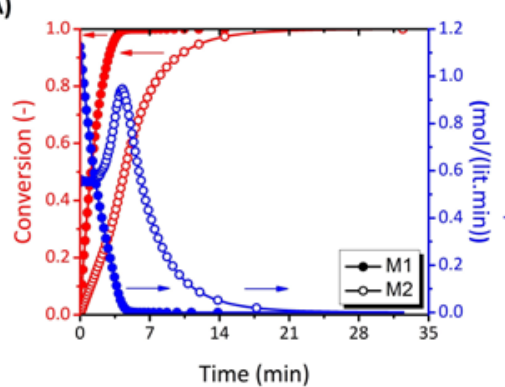

(B)

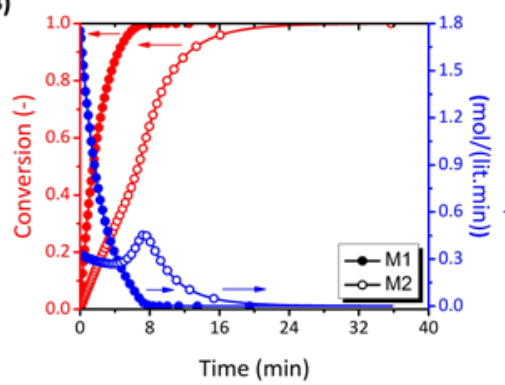

(C)

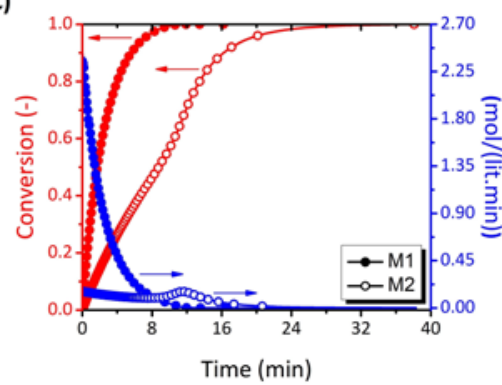

( $\left.A^{\prime}\right)$

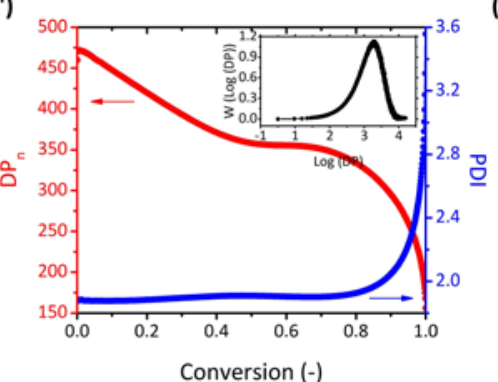

(B')

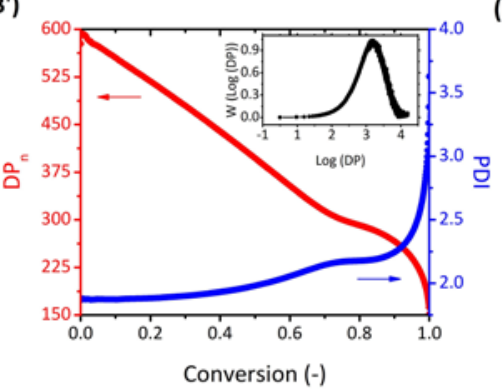

(C')

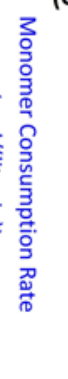

(A")

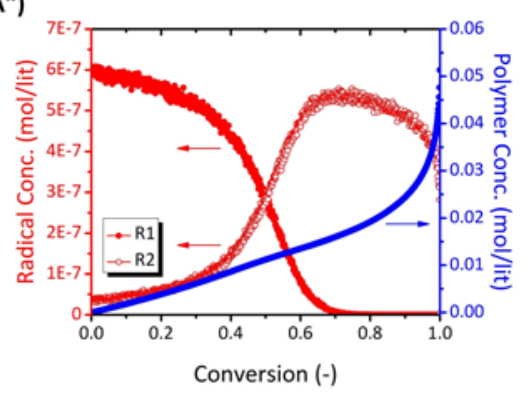

(B")

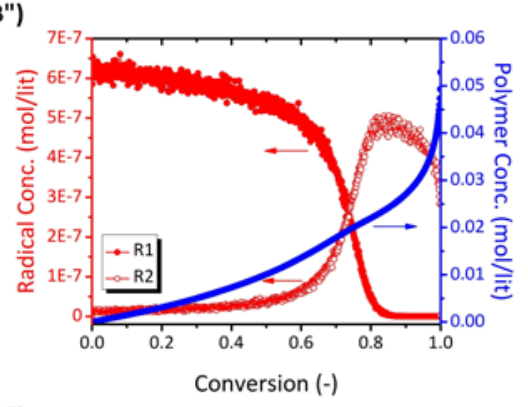

(C")

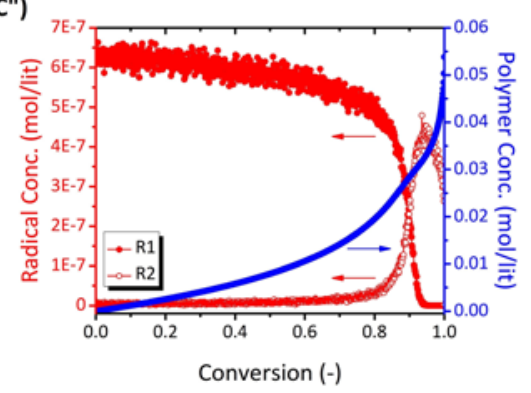

Figure 5. Profiles of monomer conversion and monomer consumption rates vs. time (A, B, C); variation of number-average degree of polymerization (DPn) and polydispersity index (PDI) of copolymer chains vs. conversion with inset of $\operatorname{CLD}\left(A^{\prime}, B^{\prime}, C^{\prime}\right)$; and variation of radical and polymer concentrations vs. conversion ( $\left.\mathrm{A}^{\prime \prime}, \mathrm{B}^{\prime \prime}, \mathrm{C}^{\prime \prime}\right) ; r_{1}=5.0$ and $r_{2}=0.1 ;\left(\mathrm{A}, \mathrm{A}^{\prime}, \mathrm{A}^{\prime \prime}\right)$ are for $f_{1}=0.25$; $\left(\mathrm{B}, \mathrm{B}^{\prime}, \mathrm{B}^{\prime \prime}\right)$ for $f_{1}=0.50$; and $\left(\mathrm{C}, \mathrm{C}^{\prime}, \mathrm{C}^{\prime \prime}\right)$ for $f_{1}=0.75$

Figure 6 shows instantaneous diad mole fractions and overall copolymer composition, F1, as a function of feed compositions vs. conversion. According to the patterns obtained by the KMC simulator for the diads (left-hand side plots in Figure 6), one can see three distinct regions, earlystage, intermediate-stage, and late-stage (Figure 6A) with respect to conversion. At the earlystage, the rate of consumption of monomer $M 1$ is higher than $M 2$; hence, diads of types 11 and 12 are dominantly formed. 
(A)

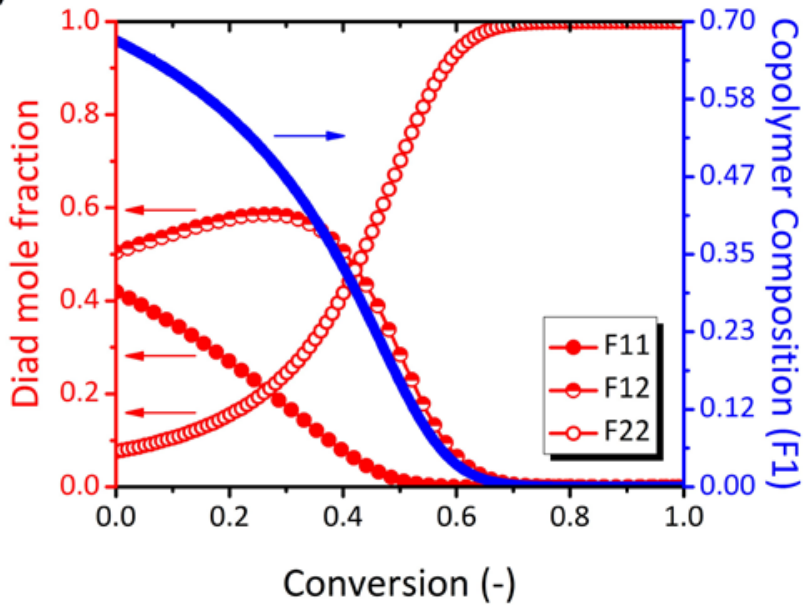

(B)

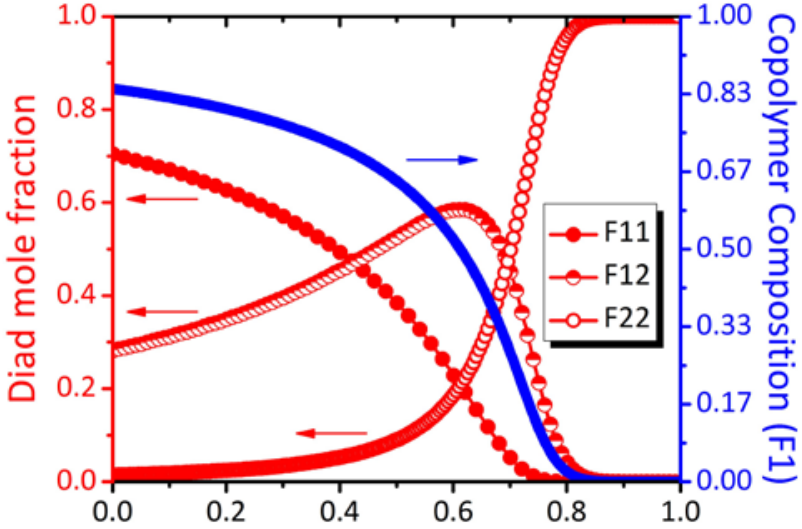

Conversion (-)

(C)

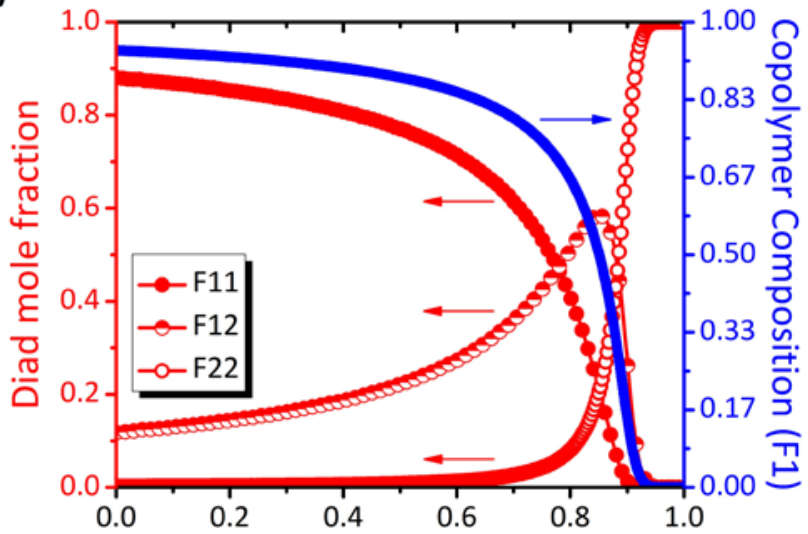

Conversion (-)
( $\left.A^{\prime}\right)$

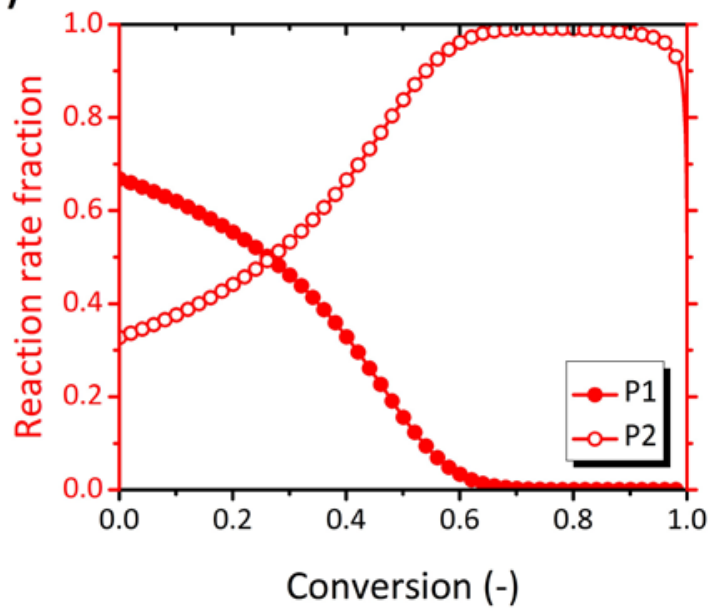

(B')

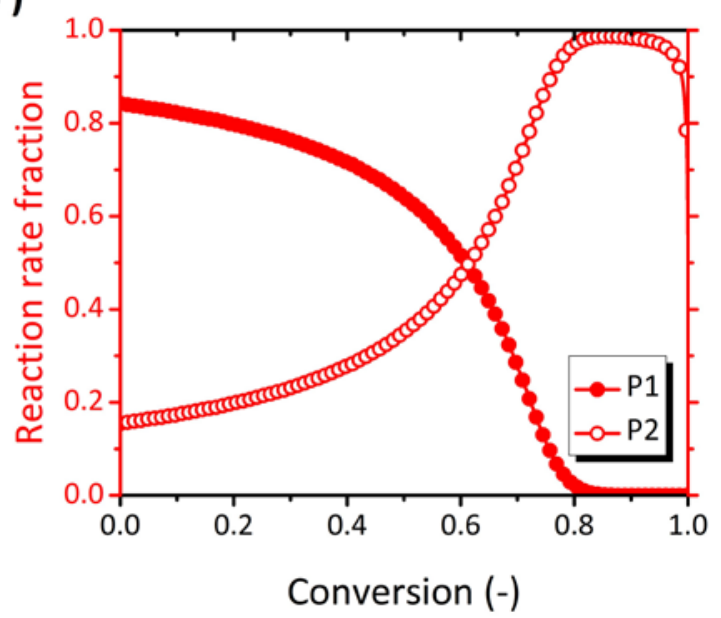

(C')

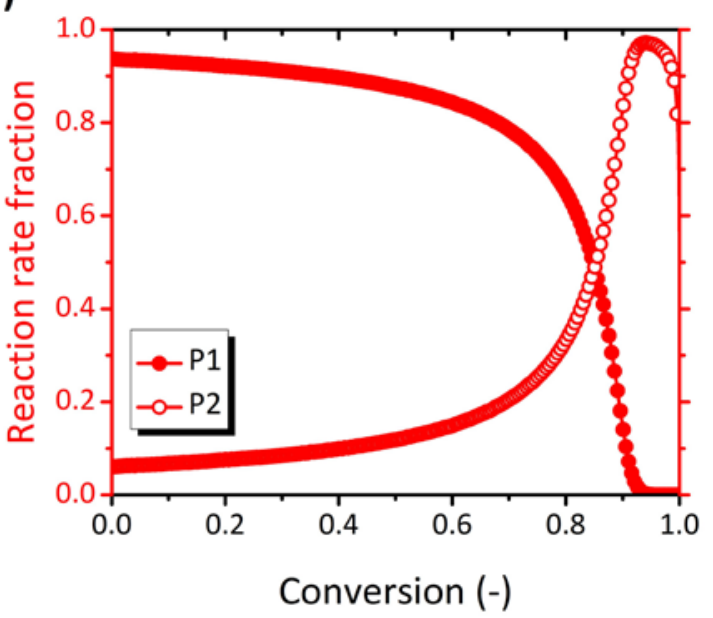

Figure 6. Profiles of instantaneous diad mole fractions (F11, F12, and F22) and instantaneous copolymer composition (F1) vs. conversion (A, B, C) and propagation reaction rate fraction (with 
$\mathrm{P} 1$ and $\mathrm{P} 2$ denoting propagating chains ending in $M 1$ and $M 2)$ vs. conversion $\left(A^{\prime}, B^{\prime}, C^{\prime}\right) ; r_{1}=5.0$ and $r_{2}=0.1 ;\left(\mathrm{A}, \mathrm{A}^{\prime}\right)$ are for $f_{1}=0.25 ;\left(\mathrm{B}, \mathrm{B}^{\prime}\right)$ for $f_{1}=0.50$; and $\left(\mathrm{C}, \mathrm{C}^{\prime \prime}\right)$ for $f_{1}=0.75$

This is consistent with the higher fraction of propagating species $M 1^{*}$ in Figure $6 A^{\prime}$. At the intermediate-stage, the amount of $M 1$ declines suddenly, monomer $M 2$ takes over, and the 12type diads are the most widespread sequence type in the polymerizing medium. Eventually, at the late-stage, where $M 1$ is consumed completely, $M 2$ controls the reaction so that only diads of the 22-type sequences can find a chance to form. It is worth mentioning that these transitions took place at higher conversion values as the content of $M 1$ in the initial feed increased.

The comparison of 2D plots obtained for the two copolymerization cases (compare Figures 1 and 2 with Figures 5 and 6 ) obviously shows more heterogeneity in the latter case, where chains with more pronounced compositional drift exist. This difference is better appreciated in 3D plots of the case with $r_{1}=5.0$ and $r_{2}=0.1$ (Figure 7 and Figure 8). Compared to the corresponding figures with $r_{1}=0.7$ and $r_{2}=0.1$ (Figure 3 and Figure 4), the variety of sequences of $M 1$ type together with steeper changes are easily observed. Also, contrasting the snapshots of distribution patterns in Figure 7 and Figure 8, one can see that at the end of polymerization, $M 2$ sequences are of limited length because of a larger contribution of $M 1$ to homo- and cross-propagation steps. The higher the percentage of $M 1$ in the initial feed, the more the variety of chains; i.e., a wider range of chain and sequence length. All these snapshots confirm that the case of $r_{1}=5.0$ and $r_{2}=0.1$ is associated with a more significant compositional drift. 
(A)

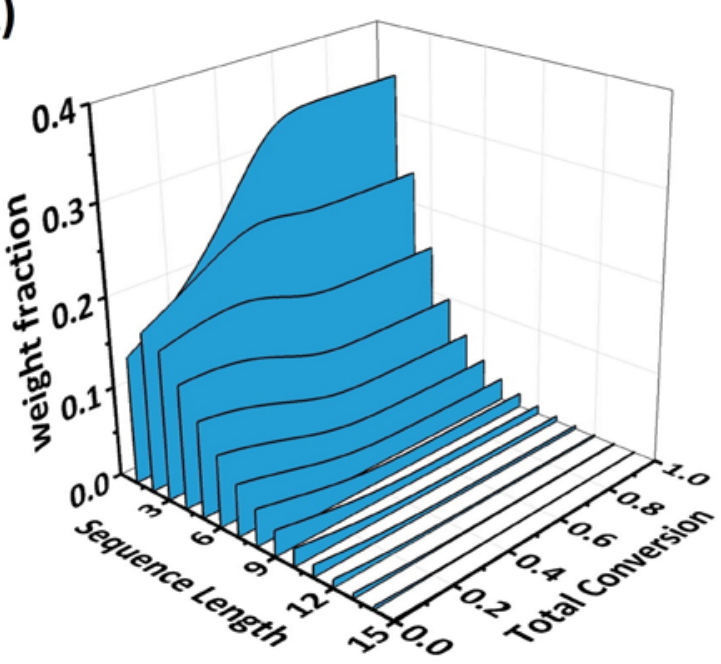

(B)

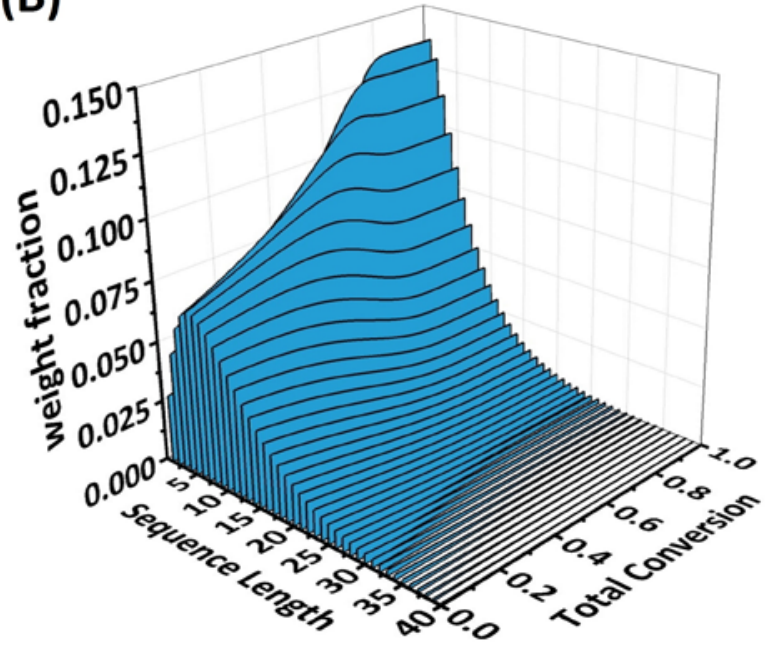

(C)

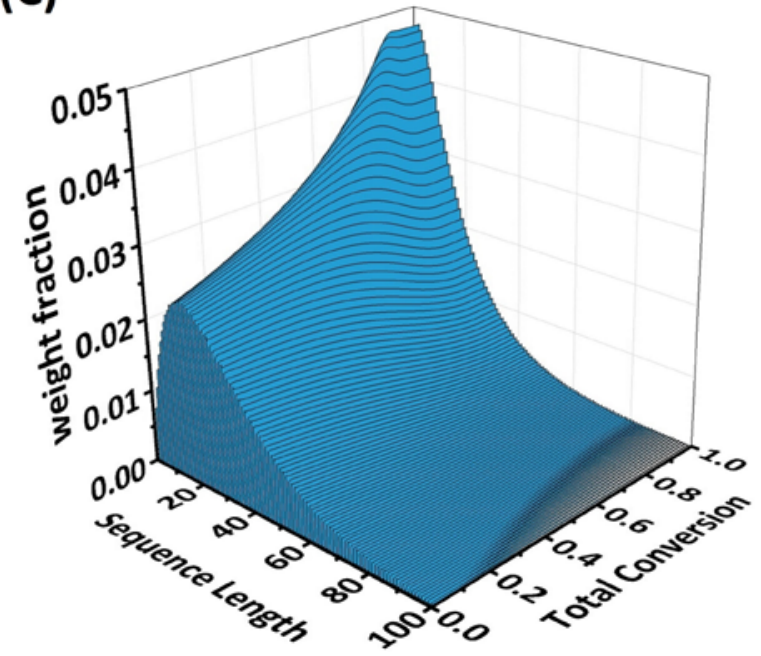

(A')

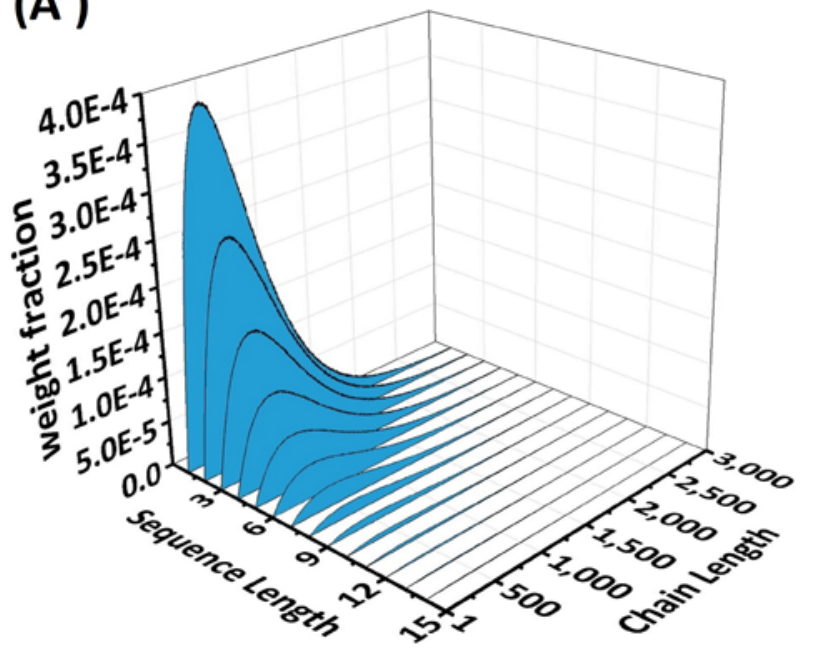

(B')

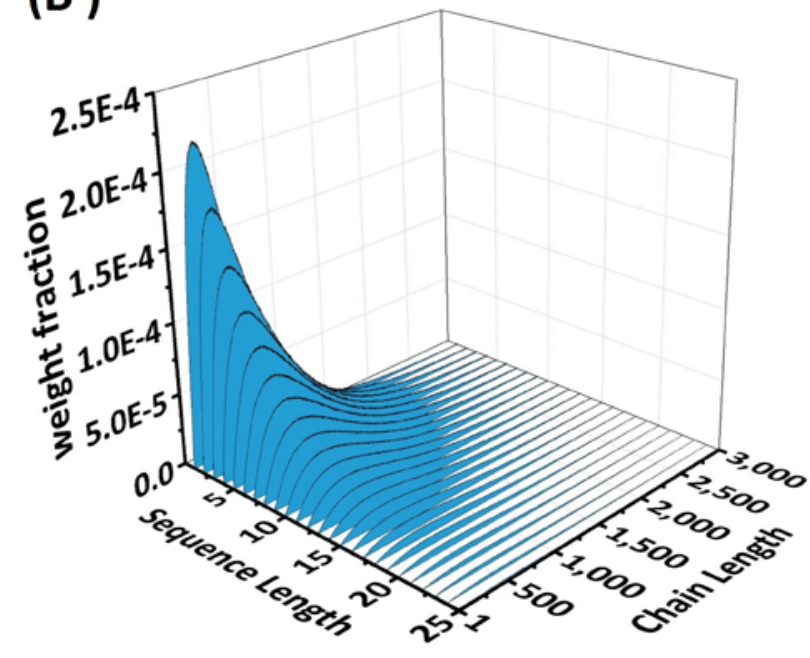

(C')

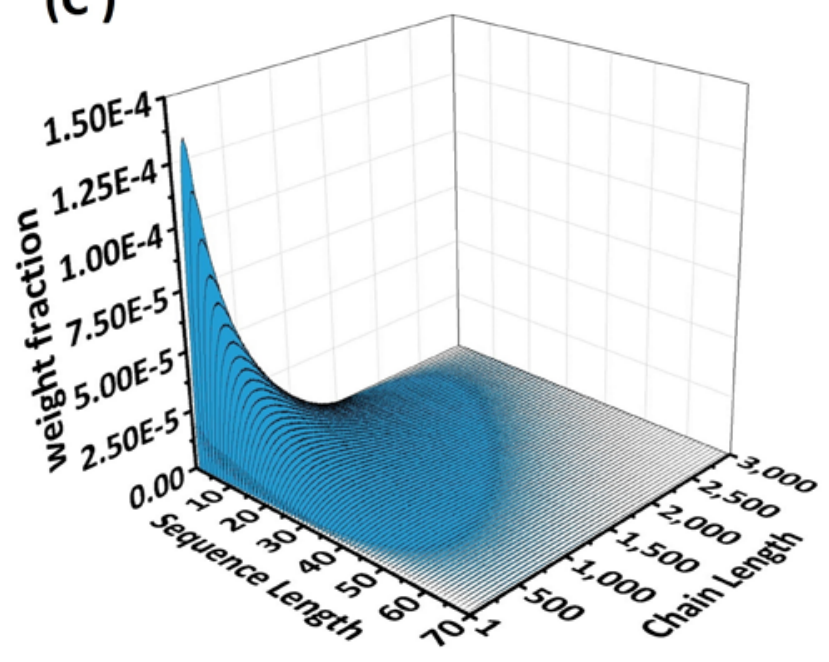


Figure 7. Profiles of evolution of weight fraction of sequence length distribution of $M 1$ under feed composition of 0.25 (A); 0.50 (B); and 0.75 (C) against conversion; and SL-CL weight distribution of $M 1$ for feed composition of $0.25\left(\mathrm{~A}^{\prime}\right) ; 0.50\left(\mathrm{~B}^{\prime}\right)$; and $0.75\left(\mathrm{C}^{\prime}\right) ; r_{1}=5.0$ and $r_{2}=0.1$ 

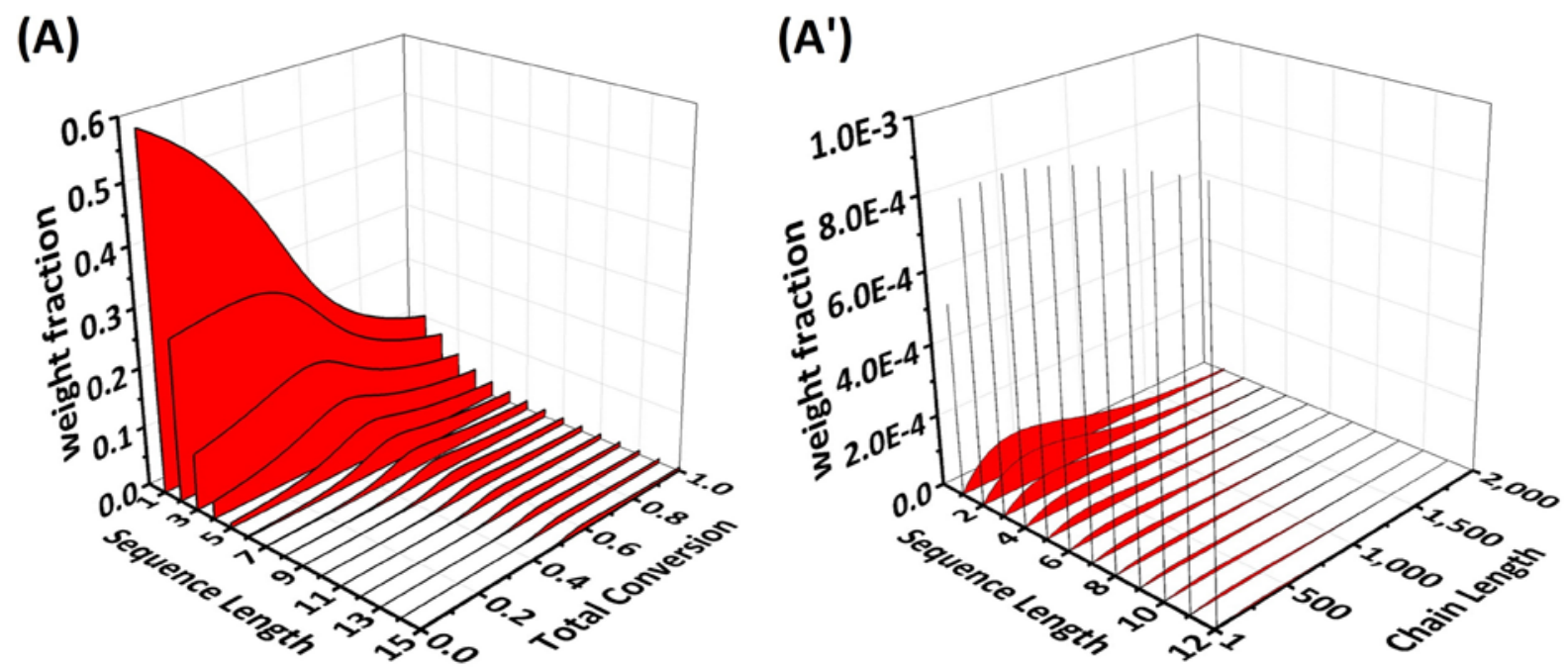

(B)

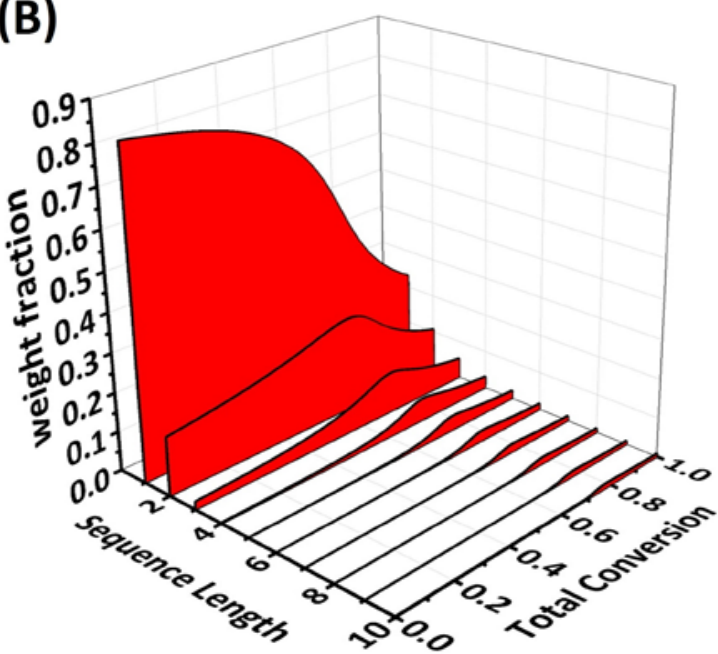

(B')

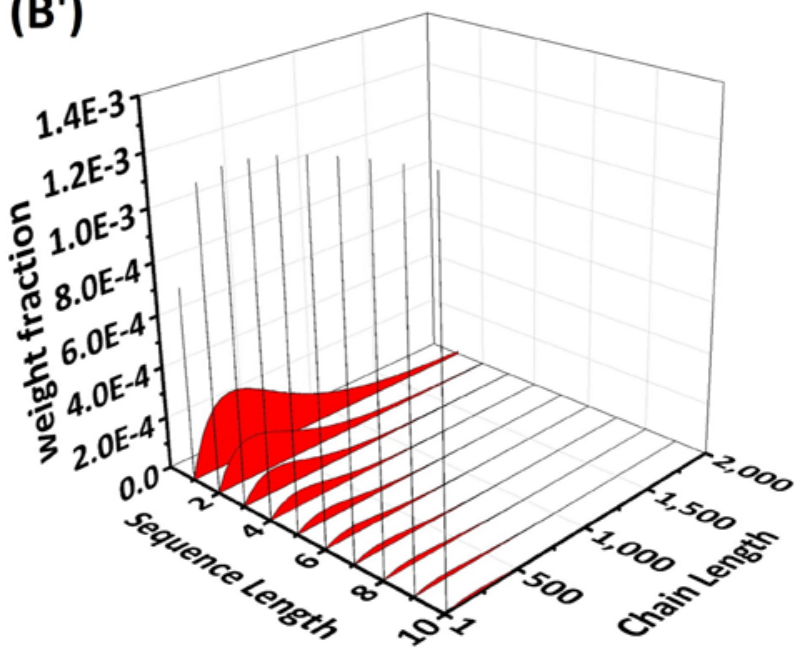

(C)

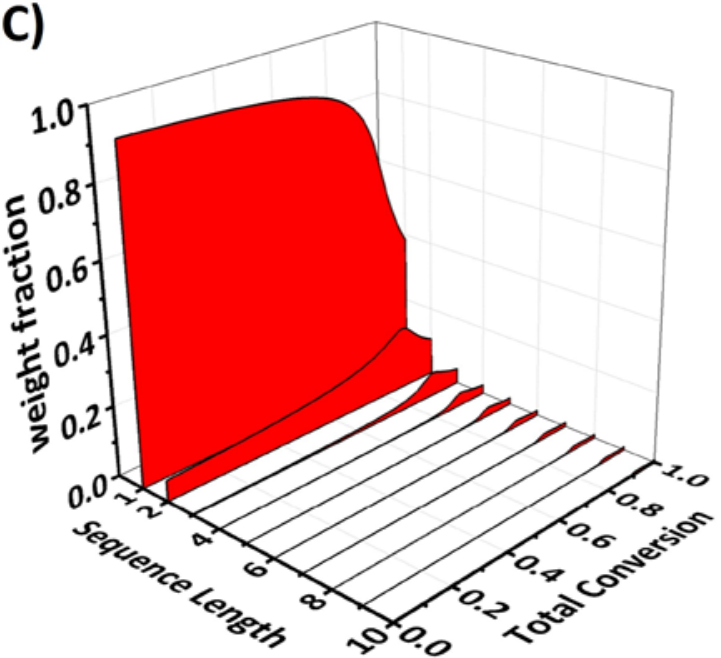

(C')

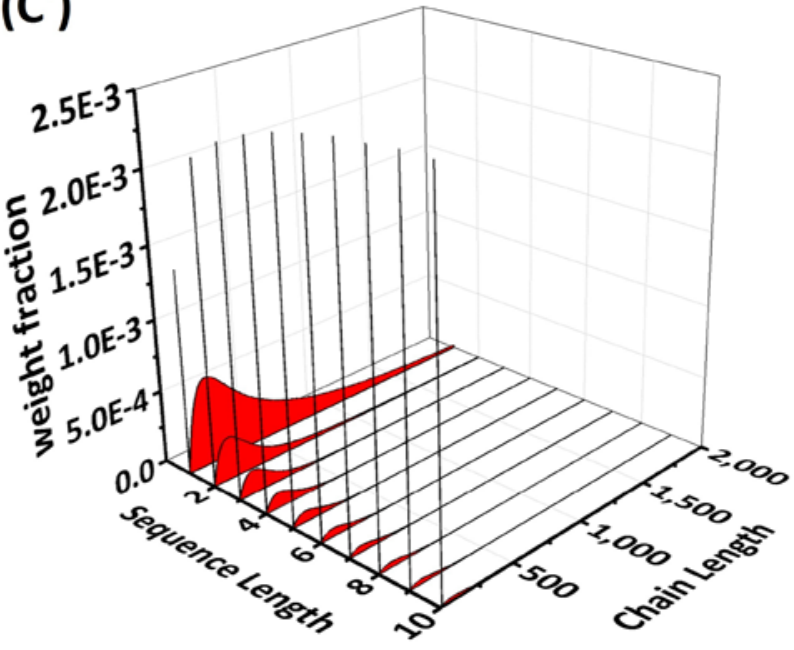


Figure 8. Profiles of evolution of weight fraction of sequence length distribution of $M 2$ under feed composition of 0.25 (A); 0.50 (B); and 0.75 (C) against conversion; and SL-CL weight distribution of $M 2$ for feed composition of $0.25\left(\mathrm{~A}^{\prime}\right) ; 0.50\left(\mathrm{~B}^{\prime}\right)$; and $0.75\left(\mathrm{C}^{\prime}\right) ; r_{1}=5.0$ and $r_{2}=0.1$

An ideal copolymerization can be treated as a special type of $r_{1}>1 \& r_{2}<1$, when $r_{1} \times r_{2}$ is equal to unity [19]. This case takes place when the two types of propagating species have the same preference for capturing monomers $M 1$ and $M 2$. Anionic and cationic copolymerizations, e.g., monomer pairs of vinylidene chloride and vinyl chloride with $r_{1}=3.2$ and $r_{2}=0.3$ are characterized by this type of behavior. The 3D plots of this type are provided with $r_{1}=2.0 \& r_{2}=0.5$ in the Appendix (Figure 13 and Figure 14). Compared to the case with $r_{1}=5.0$ and $r_{2}=0.1$ (Figure 7 and Figure 8), the reactivity ratios are closer to each other, there is a higher frequency of incorporation of both $\mathrm{M} 1$ and $\mathrm{M} 2$ to the copolymer chains, and dependency on feed composition seems more significant, with quite a remarkable presence of homopolymer chains together with copolymer ones.

\subsection{Simulation of SL-CL Distribution of Styrene-Acrylonitrile Copolymerization}

The KMC code developed in this work has been applied to the copolymerization of styrene (Sty) and acrylonitrile (AN) successfully modeled by Kiparissides et al. [23]. As mentioned earlier, the code remains flexible irrespective of reactivity ratios, composition of feed, and type of reactions involved in the scheme. Styrene-acrylonitrile (SAN) resins are among engineering thermoplastics suitable for industrial applications thanks to their load-bearing capabilities together with transparency, high heat distortion temperature, and excellent chemical resistance [24].

The reaction scheme adopted is shown in Scheme 4. It can be seen that transfer to monomer is added to the channels compared to the cases studied before (Scheme 2). The developed algorithm is able to monitor microstructural evolution, both instantaneously and cumulatively, without any limitation. Considering the data given in Table 2 and Table 3 [23], the number of initial monomer and initiator molecules were computed accordingly and fed to the simulator.
(1)
Initiator dissociation
$I \stackrel{k_{d}}{\longrightarrow} 2 f P R^{\bullet}$ 
$(2,3) \quad$ Initiation

(4-7) Propagation

(8-11) Chain transfer to monomer

$i, j: 1$ or 2 (1: Styrene (Sty) and 2: Acrylonitrile (AN))

$P R^{\bullet}$ : Primary radical

$f:$ Initiator efficiency

I , $M_{j}$ : Initiator molecule and monomer/comonomer, respectively

$R_{n, i}^{\cdot}$ : Macroradical with $n$ repeat units ending in $i$-type monomer

$P_{n}$ : Copolymer chain with $n$ repeat units

Scheme 4. Reaction scheme applied in KMC simulation of free radical copolymerization of StyAN

Table 2. Initial conditions applied to KMC simulation of free radical copolymerization of Sty-AN

\begin{tabular}{lll}
\hline Parameter & Value & Unit \\
\hline Initial initiator concentration & 0.05 & $\mathrm{~mol} \mathrm{lit}^{-1}$ \\
Molecular weight of monomer 1 & 104.14 & $\mathrm{~g} \mathrm{~mol}^{-1}$ \\
Molecular weight of monomer 2 & 53.06 & $\mathrm{~g} \mathrm{~mol}^{-1}$ \\
Density of monomer 1 & 0.87 & $\mathrm{~g} \mathrm{~cm}^{-3}$ \\
Density of monomer 2 & 0.76 & $\mathrm{~g} \mathrm{~cm}^{-3}$ \\
Density of copolymer 1 & 1.05 & $\mathrm{~g} \mathrm{~cm}^{-3}$ \\
Density of copolymer 2 & 1.15 & $\mathrm{~g} \mathrm{~cm}^{-3}$ \\
Copolymerization temperature & 60 & ${ }^{\circ} \mathrm{C}$ \\
\hline
\end{tabular}

Table 3. Kinetic parameters used in KMC simulation of free radical copolymerization of Sty (monomer 1) and AN (monomer 2)

\begin{tabular}{|c|c|c|}
\hline Parameter & Value & Unit \\
\hline Initiator dissociation rate constant $\left(\boldsymbol{k}_{d}\right)$ & $8.1217 \times 10^{-6}$ & $\sec ^{-1}$ \\
\hline Initiator efficiency $(f)$ & 0.58 & - \\
\hline Initiation rate constant $\left(\boldsymbol{k}_{1}\right)$ & $2.5821 \times 10^{2}$ & lit $\mathrm{mol}^{-1} \mathrm{sec}^{-1}$ \\
\hline Initiation rate constant $\left(\boldsymbol{k}_{\mathbf{2}}\right)$ & $4.1402 \times 10^{5}$ & lit $\mathrm{mol}^{-1} \mathrm{sec}^{-1}$ \\
\hline Homo-propagation rate constant (Sty, $\left.\boldsymbol{k}_{p, 11}\right)$ & $2.5821 \times 10^{2}$ & lit $\mathrm{mol}^{-1} \mathrm{sec}^{-1}$ \\
\hline Homo-propagation rate constant (AN, $\boldsymbol{k}_{p, 22}$ ) & $4.1402 \times 10^{5}$ & lit $\mathrm{mol}^{-1} \mathrm{sec}^{-1}$ \\
\hline Reactivity ratio of Monomer $1\left(r_{1}\right)$ & 0.36 & - \\
\hline Reactivity ratio of Monomer $2\left(r_{2}\right)$ & 0.078 & - \\
\hline Chain transfer to monomer rate constant $\left(\boldsymbol{k}_{t M, 11}\right)$ & $1.1226 \times 10^{-2}$ & lit $\mathrm{mol}^{-1} \mathrm{sec}^{-1}$ \\
\hline
\end{tabular}


Chain transfer to monomer rate constant $\left(\boldsymbol{k}_{t M, 12}\right) \quad 3.3759 \times 10^{-1} \quad$ lit mol $\mathrm{m}^{-1} \mathrm{sec}^{-1}$ Chain transfer to monomer rate constant $\left(\boldsymbol{k}_{t M, 22}\right) \quad 6.8432 \quad$ lit mol ${ }^{-1} \mathrm{sec}^{-1}$

Chain transfer to monomer rate constant $\left(\boldsymbol{k}_{t M, 21}\right) \quad 3.4092 \times 10^{1} \quad$ lit mol-1 $\mathrm{sec}^{-1}$

Termination by combination rate constant $\left(\boldsymbol{k}_{t c, 11}\right) \quad 9.9663 \times 10^{7} \quad$ lit $\mathrm{mol}^{-1} \mathrm{sec}^{-1}$

Termination by combination rate constant $\left(\boldsymbol{k}_{t c, 22}\right) \quad 9.4650 \times 10^{8} \quad \mathrm{lit} \mathrm{mol}^{-1} \mathrm{sec}^{-1}$

Termination by combination rate constant $\left(\boldsymbol{k}_{t c, 12}\right) \quad 1.8458 \times 10^{9} \quad$ lit mol $^{-1} \mathrm{sec}^{-1}$

SAN copolymerization is associated with random placement of monomers along chains leading to linear amorphous copolymer chains. Normally, physical properties and processability of SAN copolymers are dependent on molecular weight and acrylonitrile content. For instance, hardness of SAN increases by increasing the AN level [24]. Thus, visualization of SL-CL distribution patterns as a function of feed composition would be very useful. A careful view of Figure $9 \mathrm{~A}$ indicates that cross-propagation should be dominant at feed composition of 0.25 , because the sequence length does not exceed the value of 5 here. At conversions higher than 0.50 , where $M 1$ is possibly consumed, $M 2$ sequences of longer length are formed (Figure 10A). This trend has been followed at initial feed composition of 0.50 with a more random character, as realized from the rectangular shape of distributions (Figure 9B and Figure 10B). At the highest feed composition studied in this work, cross- and homo-polymerization took place at the same time. Diversity of chains together with formation of homopolymer chains, featured via the abundance of short sequences, is detectable in Figure $9 \mathrm{C}$ and Figure $9 \mathrm{C}^{\prime}$. It can be realized from the SL-CL distribution patterns that the frequency and length of sequences have markedly been governed by the feed composition. 
(A)

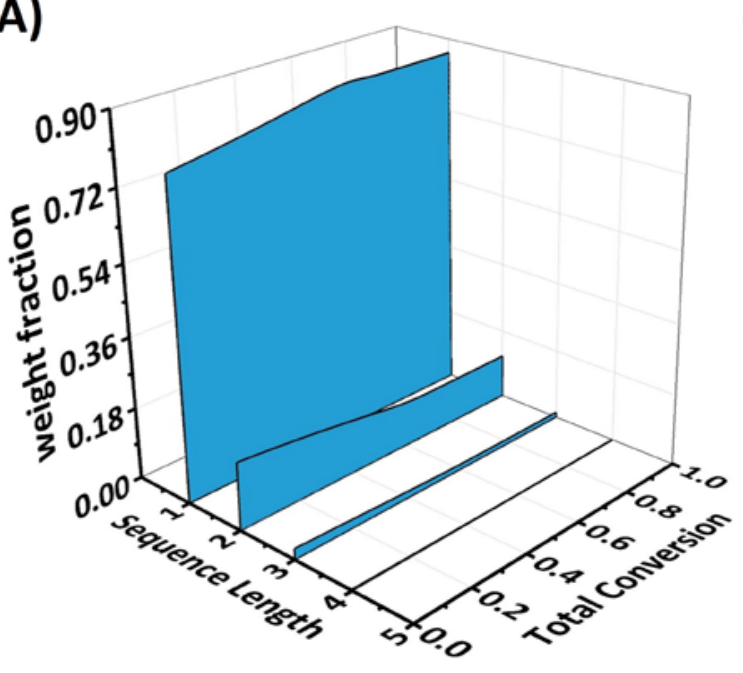

(B)

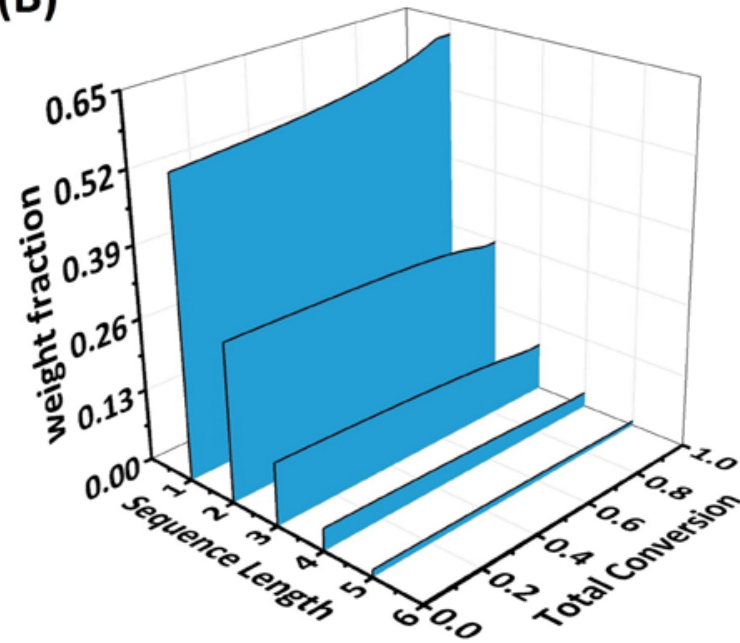

(C)

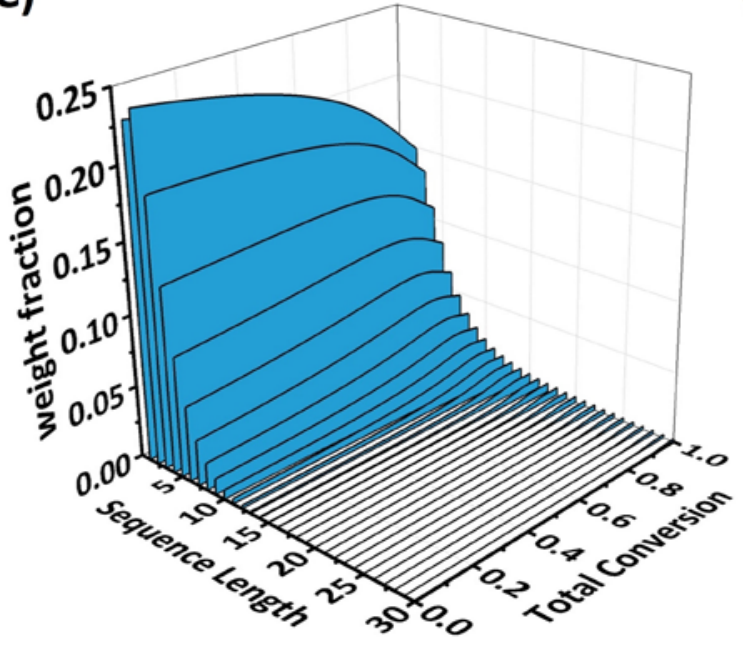

(A')

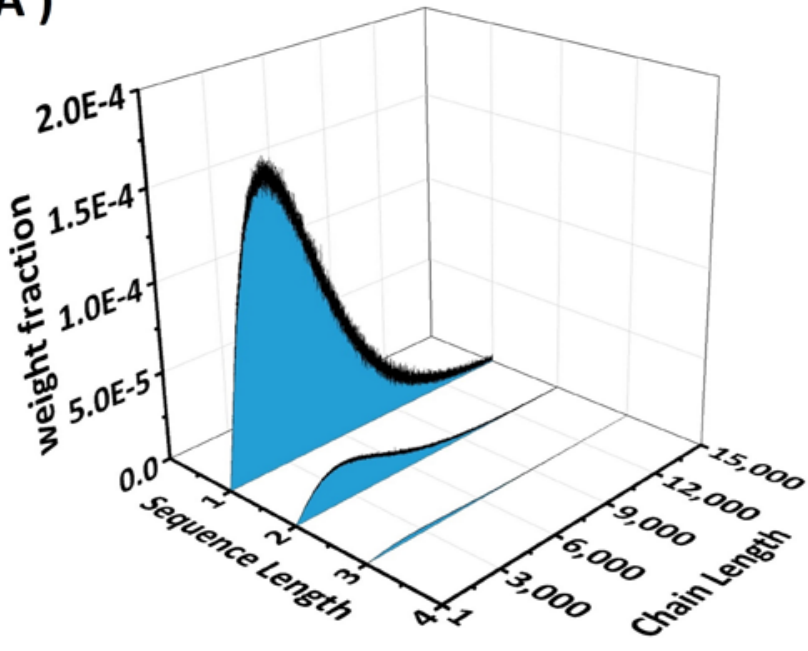

(B')

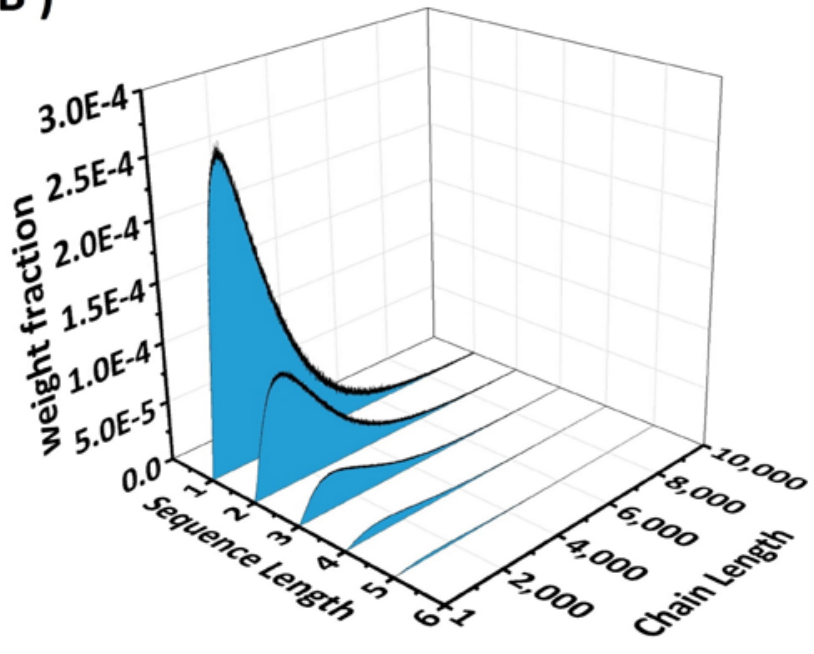

(C')

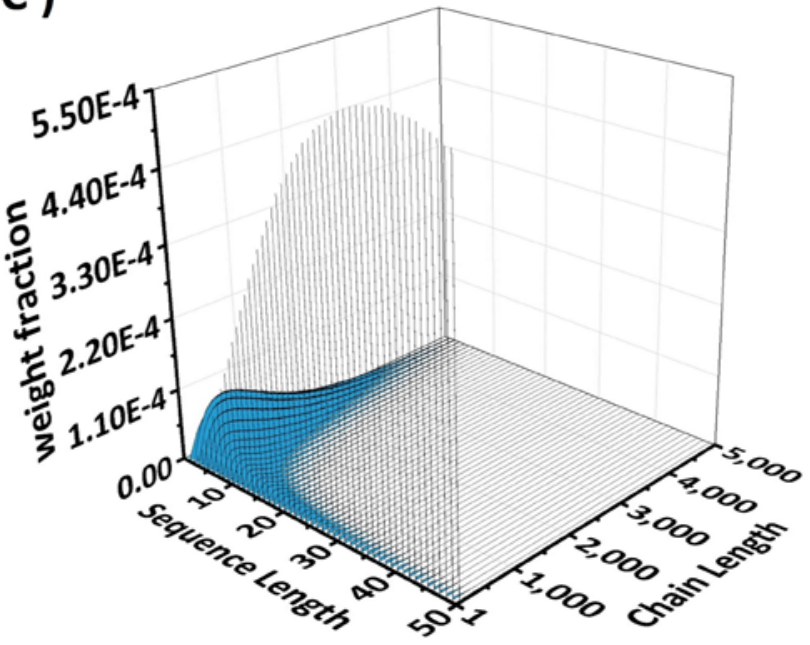


Figure 9. Profiles of evolution of weight fraction of sequence length distribution of Sty ( $M 1)$ in copolymerization with AN (M2) with $r_{1}=0.36$ and $r_{2}=0.078$ under feed composition of 0.25 (A); 0.50 (B); and 0.75 (C) against conversion; and SL-CL weight distribution of Sty for feed composition of $0.25\left(A^{\prime}\right) ; 0.50\left(B^{\prime}\right)$; and $0.75\left(C^{\prime}\right)$ 
(A)

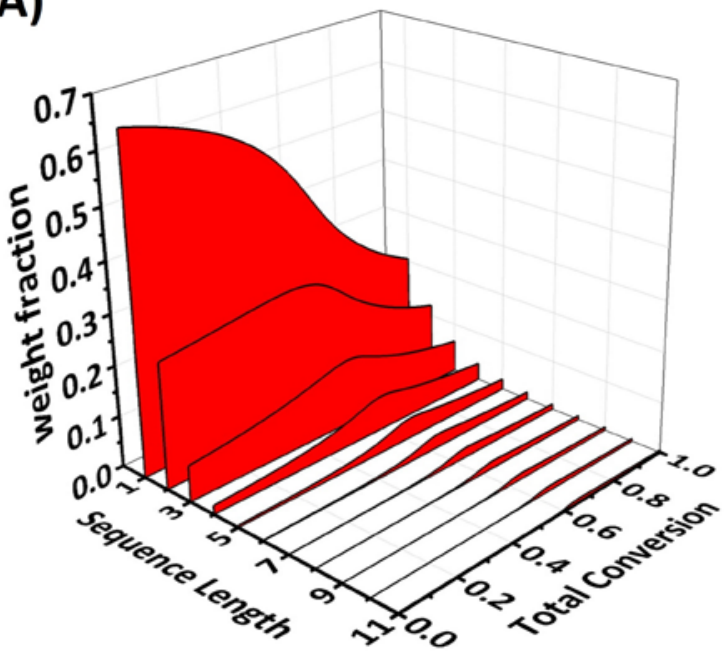

(B)

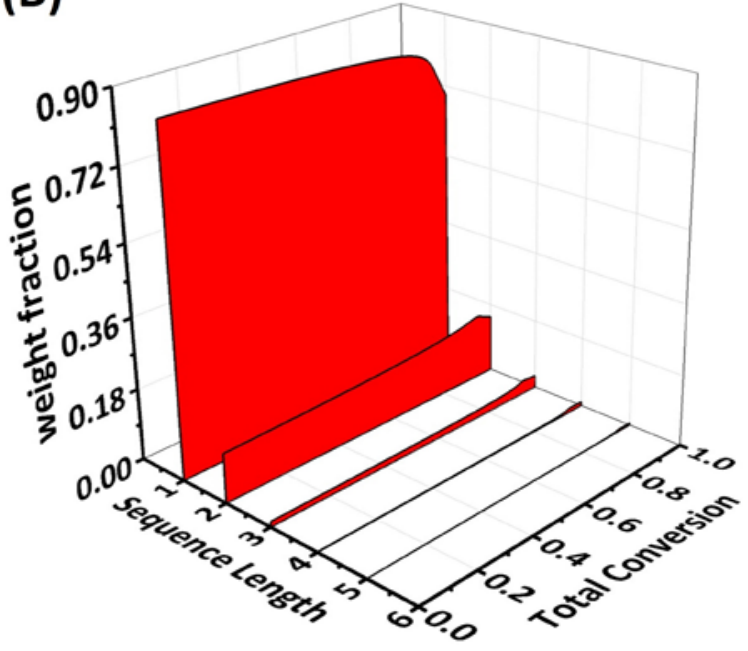

(C)

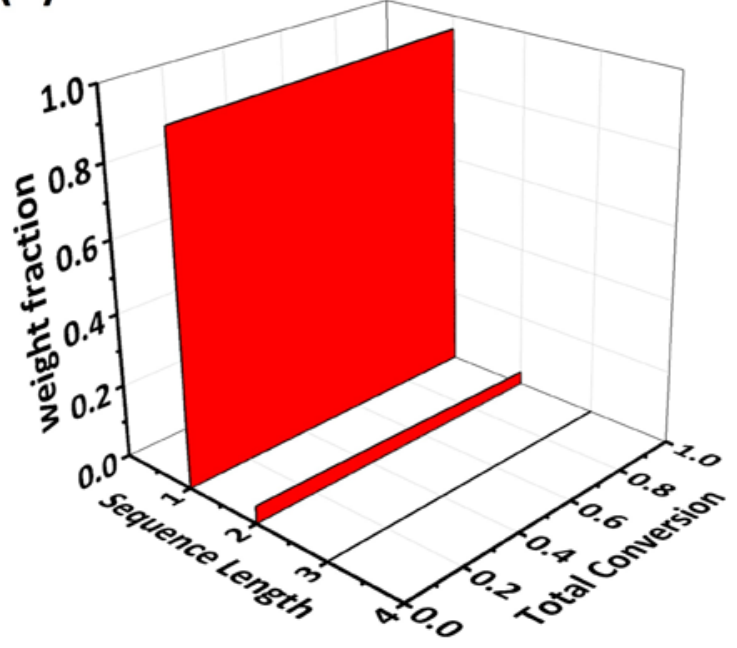

(A')

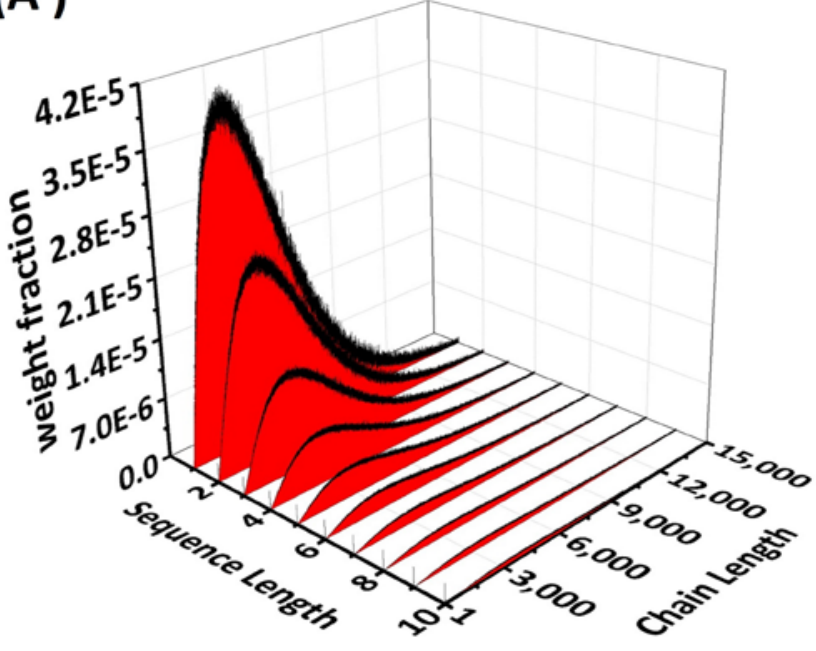

(B')

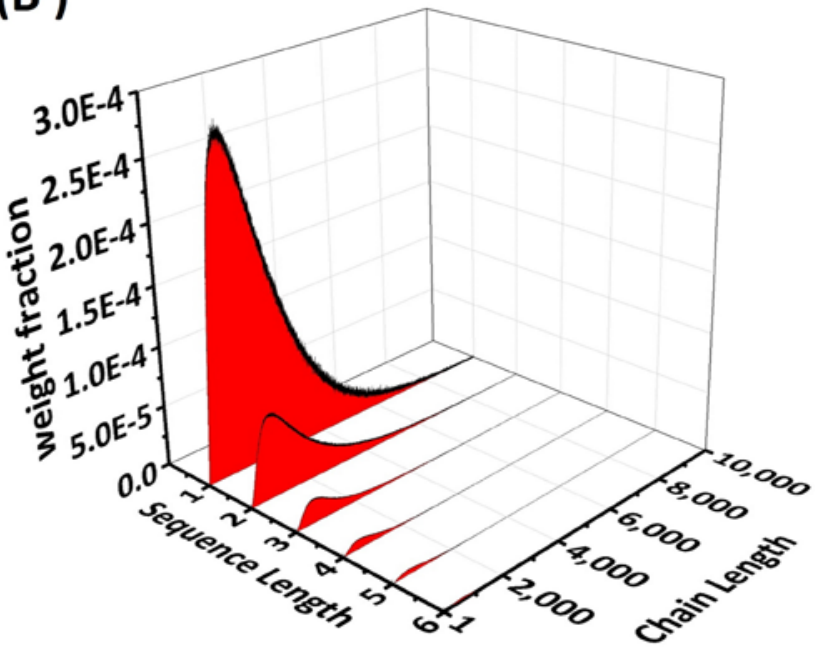

(C')

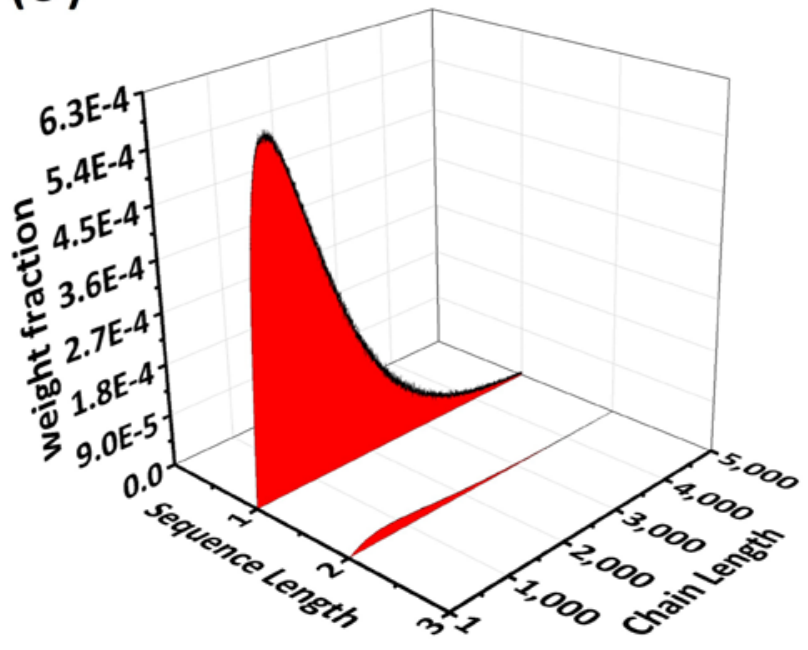


Figure 10. Profiles of evolution of weight fraction of sequence length distribution of $A N(M 2)$ in copolymerization with Sty (M1) with $r_{1}=0.36$ and $r_{2}=0.078$ under feed composition of $0.25(\mathrm{~A})$;

0.50 (B); and 0.75 (C) against conversion; and SL-CL weight distribution of $A N$ for feed composition of $0.25\left(\mathrm{~A}^{\prime}\right)$; $0.50\left(\mathrm{~B}^{\prime}\right)$; and $0.75\left(\mathrm{C}^{\prime}\right)$

\section{CONCLUSION}

A versatile Kinetic Monte Carlo (KMC) code has been developed and applied to the simulation of free radical copolymerization of monomers of different reactivity ratios ( $r_{1}$ and $\left.r_{2}\right)$ under various feed compositions. The code successfully monitored the microstructural evolution during the course and at the end of the polymerization and made it possible to explicitly capture, screen (based on conversion, chain length, and sequence length), and visualize the bivariate dynamic sequence length-conversion and end-of-batch sequence length-chain length distribution for different types of copolymers with reactivity ratios of $\left(r_{1}<1 \& r_{2}<1\right) ;\left(r_{1}>1 \& r_{2}<1\right) ;\left(r_{1} \times r_{2}=1\right)$; and $\left(r_{1}=r_{2}=1\right)$. Typical bivariate distribution patterns were plotted for either $M 1$ or $M 2$ monomers for feeds composed of 25,50 , and 75 mole percent of $M 1$, based on a typical polymerization scheme considering initiation, homo- and cross-propagation, and termination (either combination or disproportionation) channels. The versatile code (easily expandable to include additional steps in the copolymerization scheme, as illustrated via the last case study) developed in this work is characterized by a powerful data storage structure with the capability of recording, sorting, and distinguishing macromolecules based on the length and position of sequences of each type extracted from every single chain in the system. The results of this work provide detailed snapshots during copolymerization, very useful for visualizing and tailor-making macromolecular chains.

\section{APPENDIX}


(A)

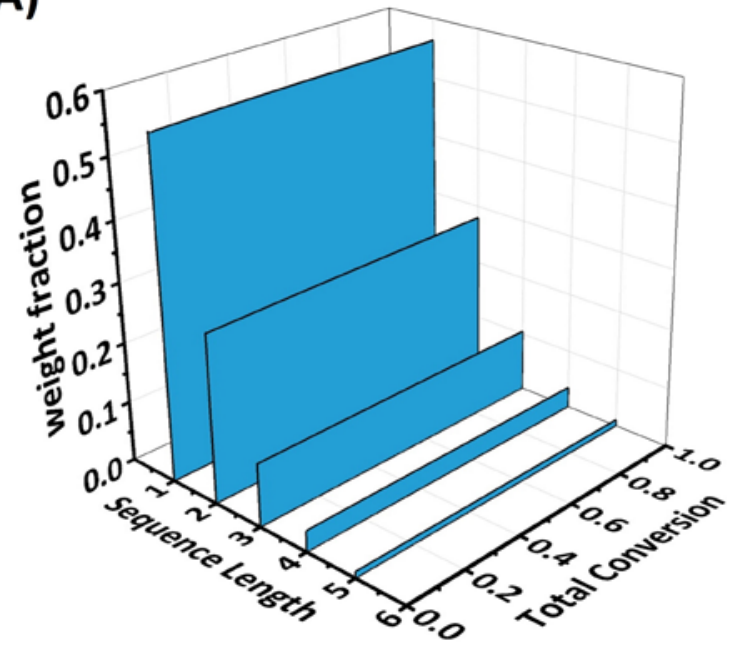

(B)

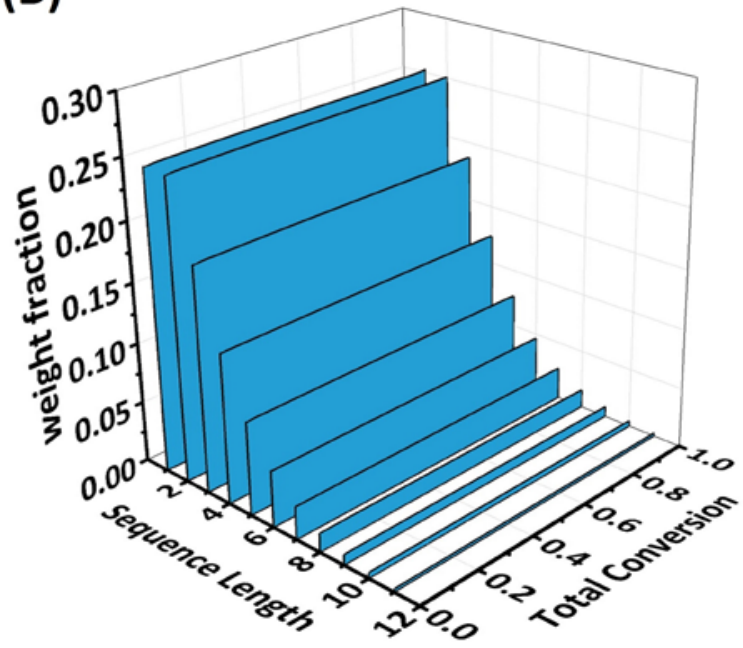

(C)

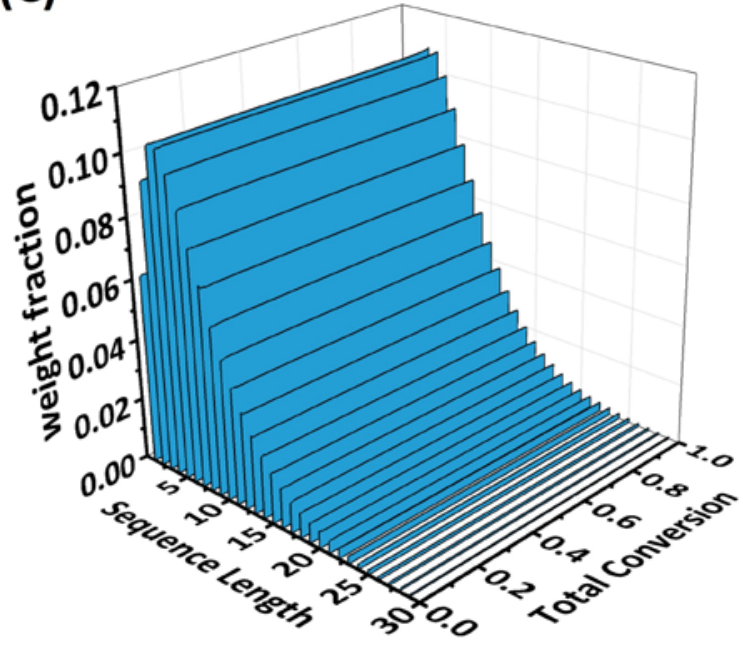

(A')

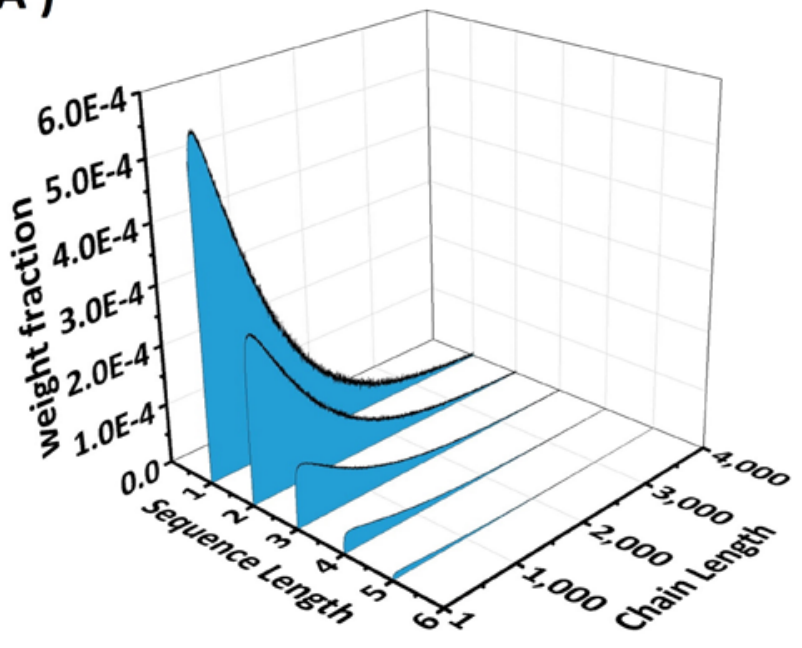

(B')

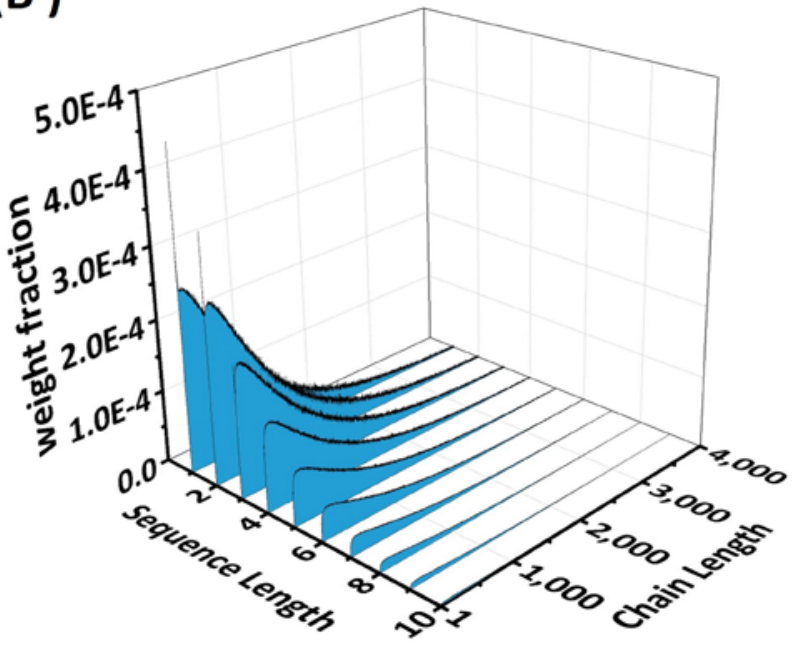

(C')

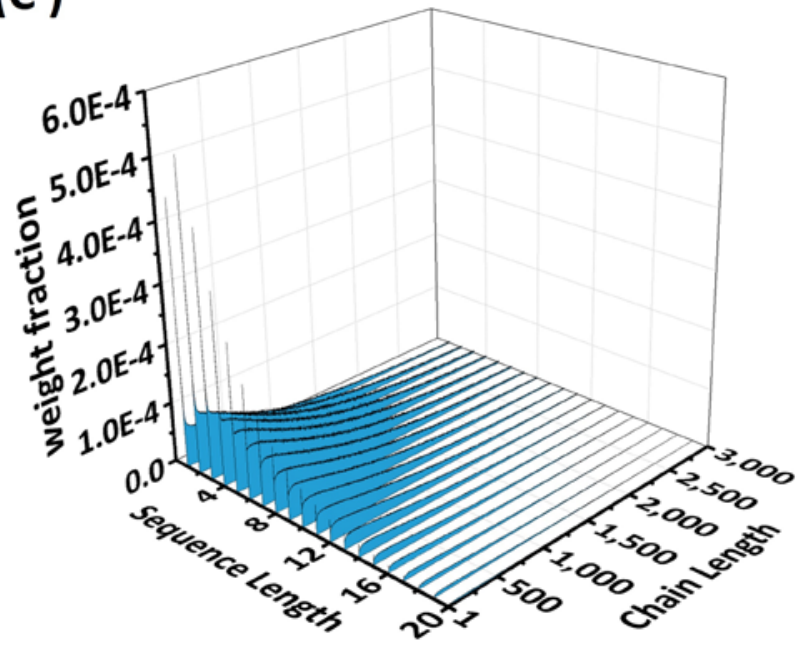


Figure 11. Profiles of evolution of weight fraction of sequence length distribution of $M 1$ under feed composition of 0.25 (A); 0.50 (B); and 0.75 (C) against conversion; and SL-CL weight distribution of $M 1$ for feed composition of $0.25\left(A^{\prime}\right) ; 0.50\left(B^{\prime}\right)$; and $0.75\left(C^{\prime}\right) ; r_{1}=1.0$ and $r_{2}=1.0$ 

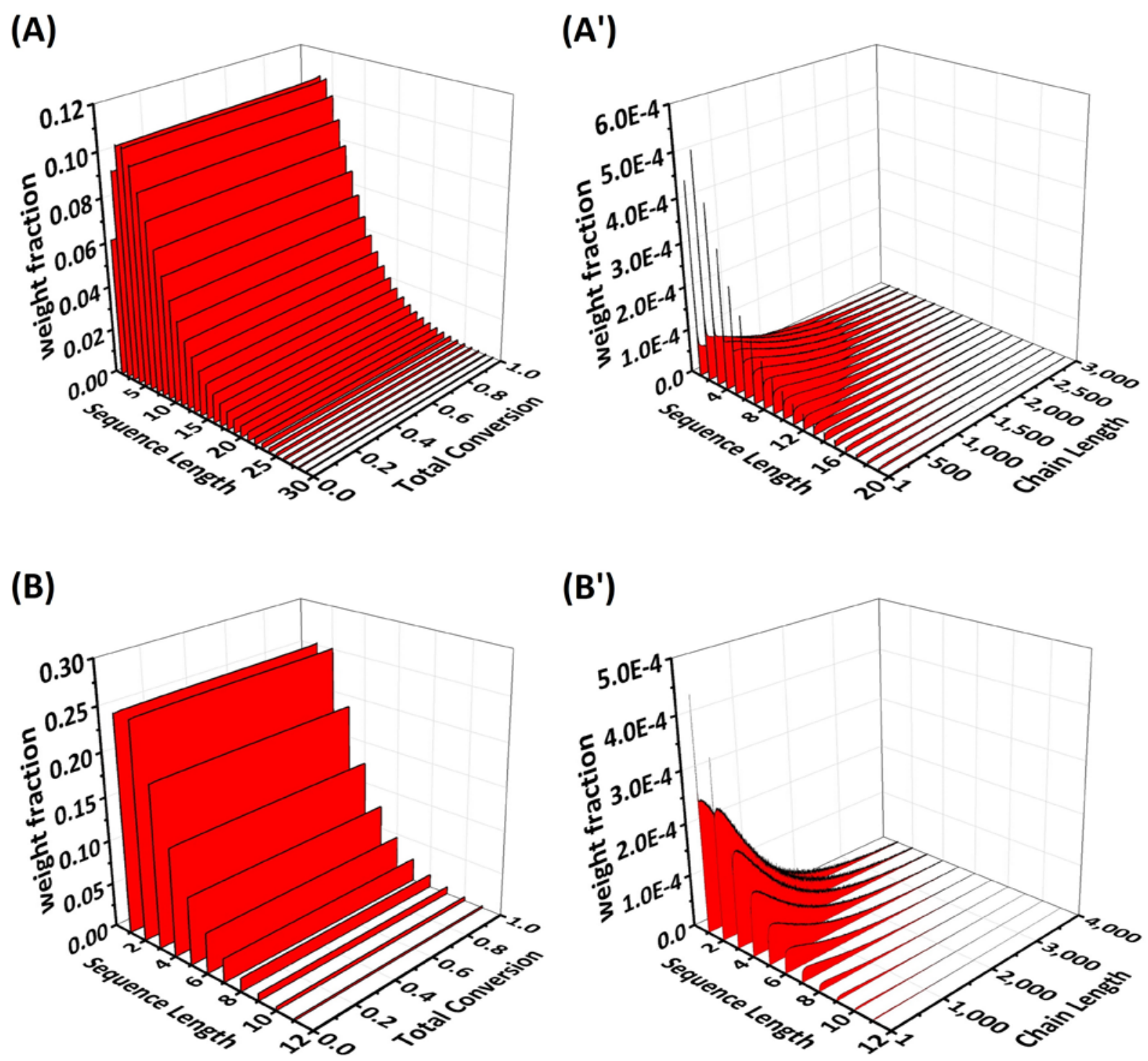

(C)

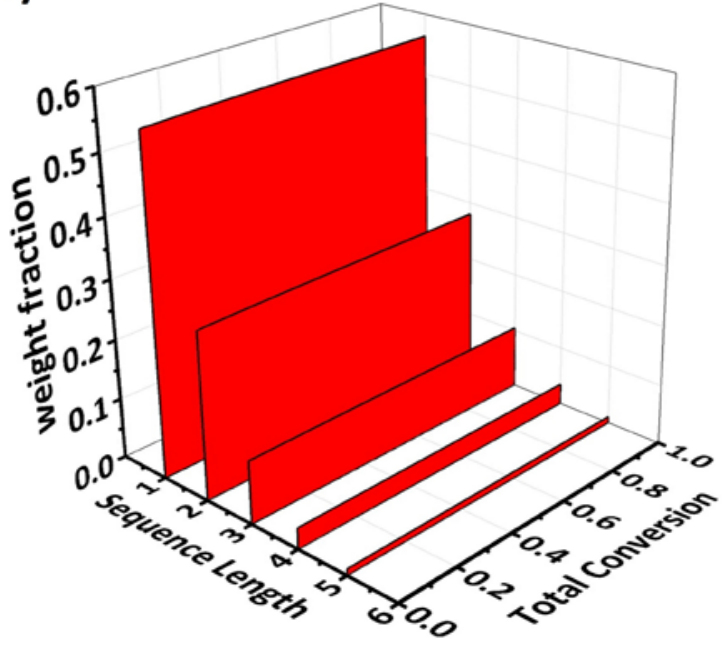

(C')

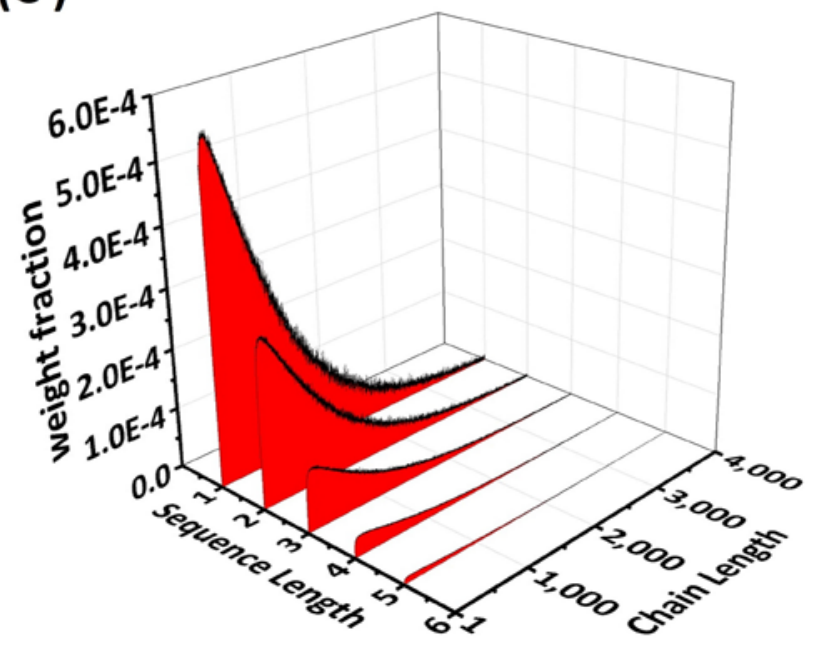


Figure 12. Profiles of evolution of weight fraction of sequence length distribution of $M 2$ under feed composition of 0.25 (A); 0.50 (B); and 0.75 (C) against conversion; and SL-CL weight distribution of $M 2$ for feed composition of $0.25\left(A^{\prime}\right) ; 0.50\left(B^{\prime}\right)$; and $0.75\left(C^{\prime}\right) ; r_{1}=1.0$ and $r_{2}=1.0$ 
(A)

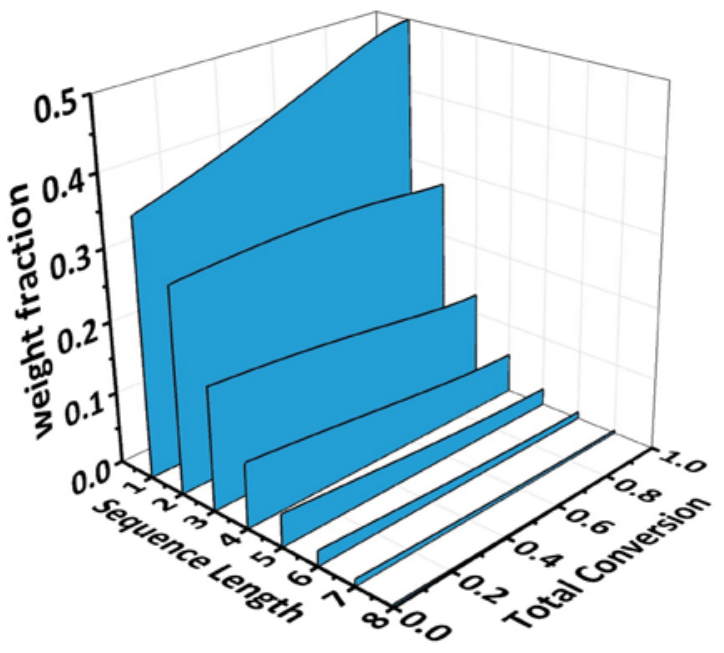

(B)

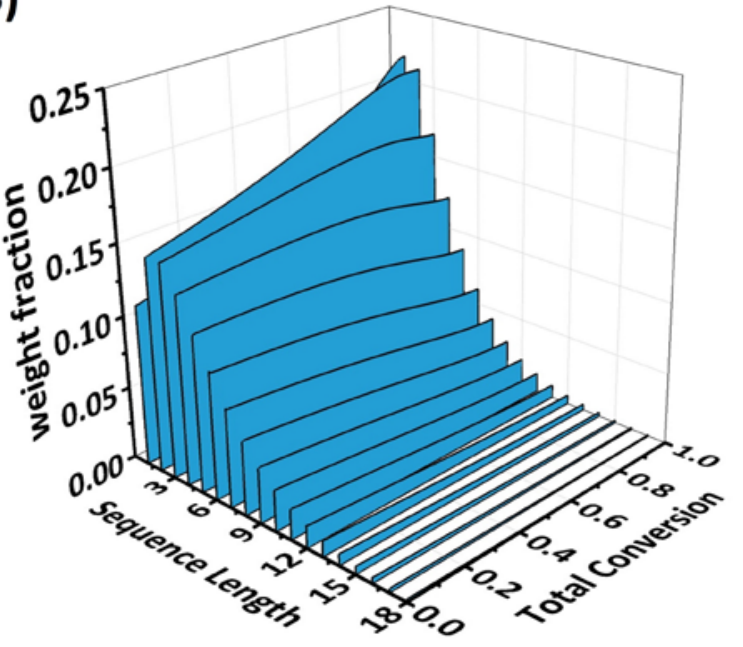

(C)

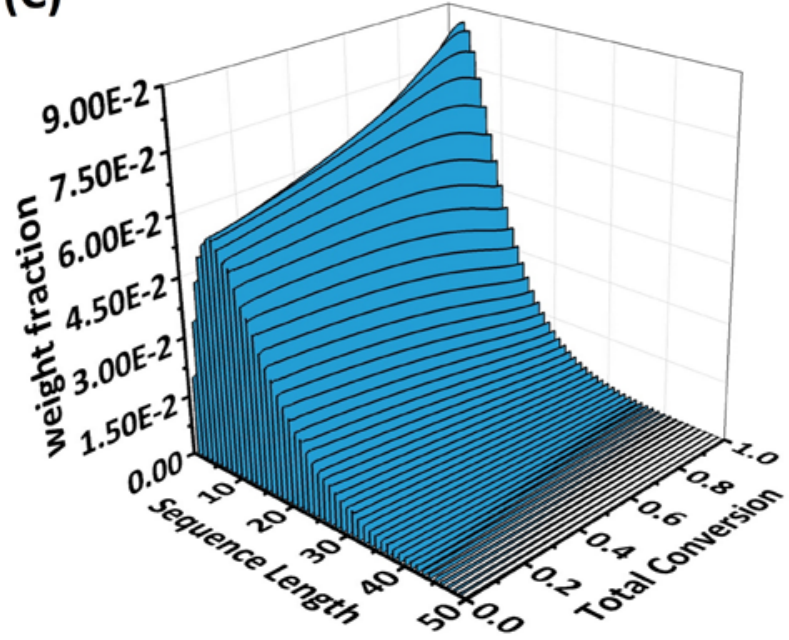

(A')

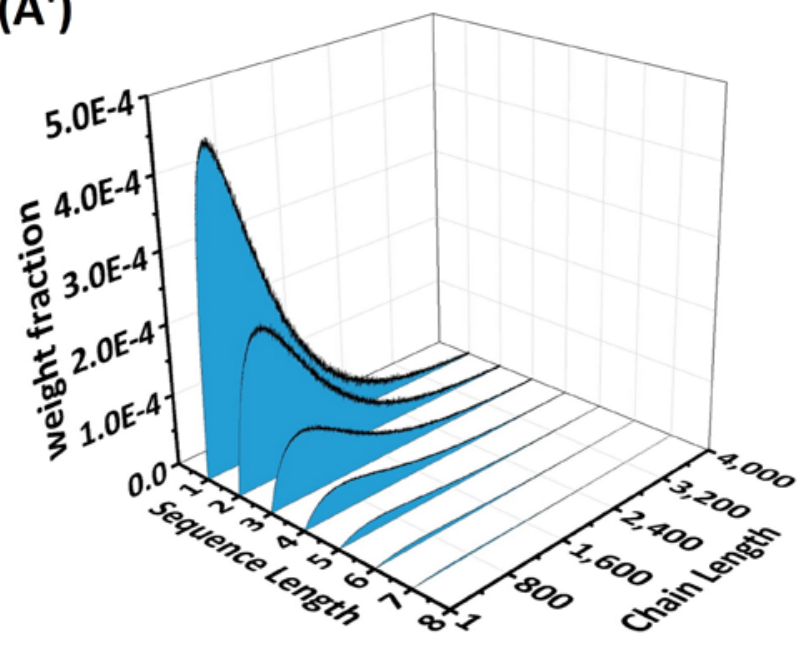

(B')

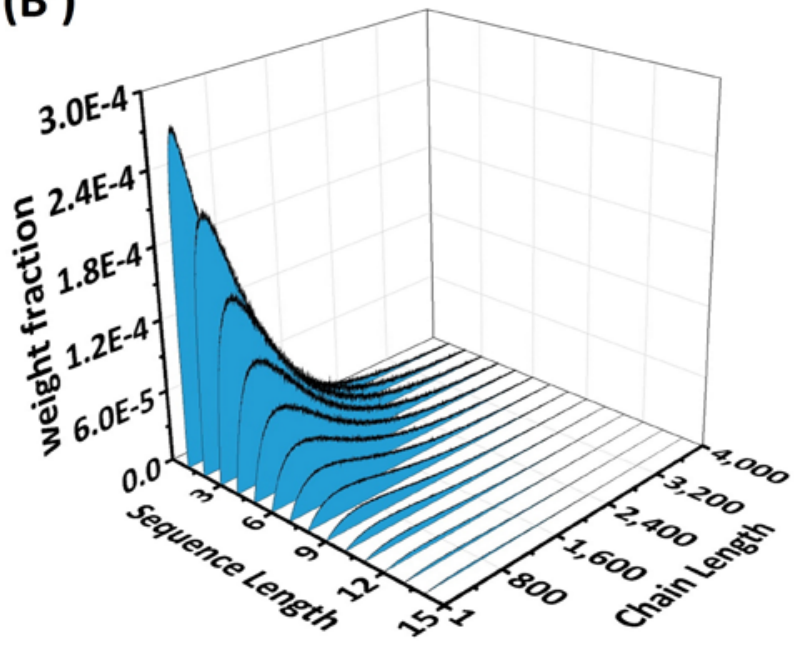

(C')

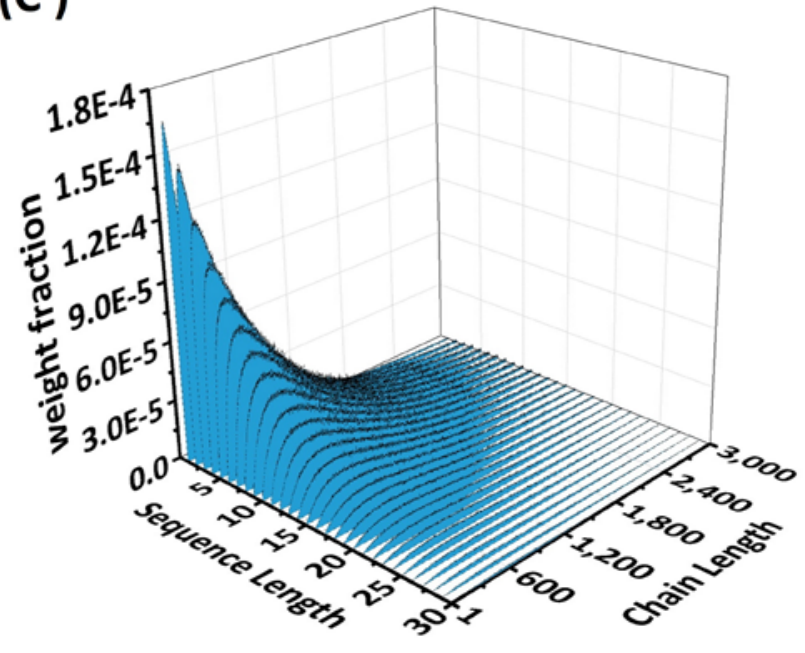


Figure 13. Profiles of evolution of weight fraction of sequence length distribution of $M 1$ under feed composition of 0.25 (A); 0.50 (B); and 0.75 (C) against conversion; and SL-CL weight distribution of $M 1$ for feed composition of $0.25\left(A^{\prime}\right) ; 0.50\left(B^{\prime}\right)$; and $0.75\left(C^{\prime}\right) ; r_{1}=2.0$ and $r_{2}=0.5$ 

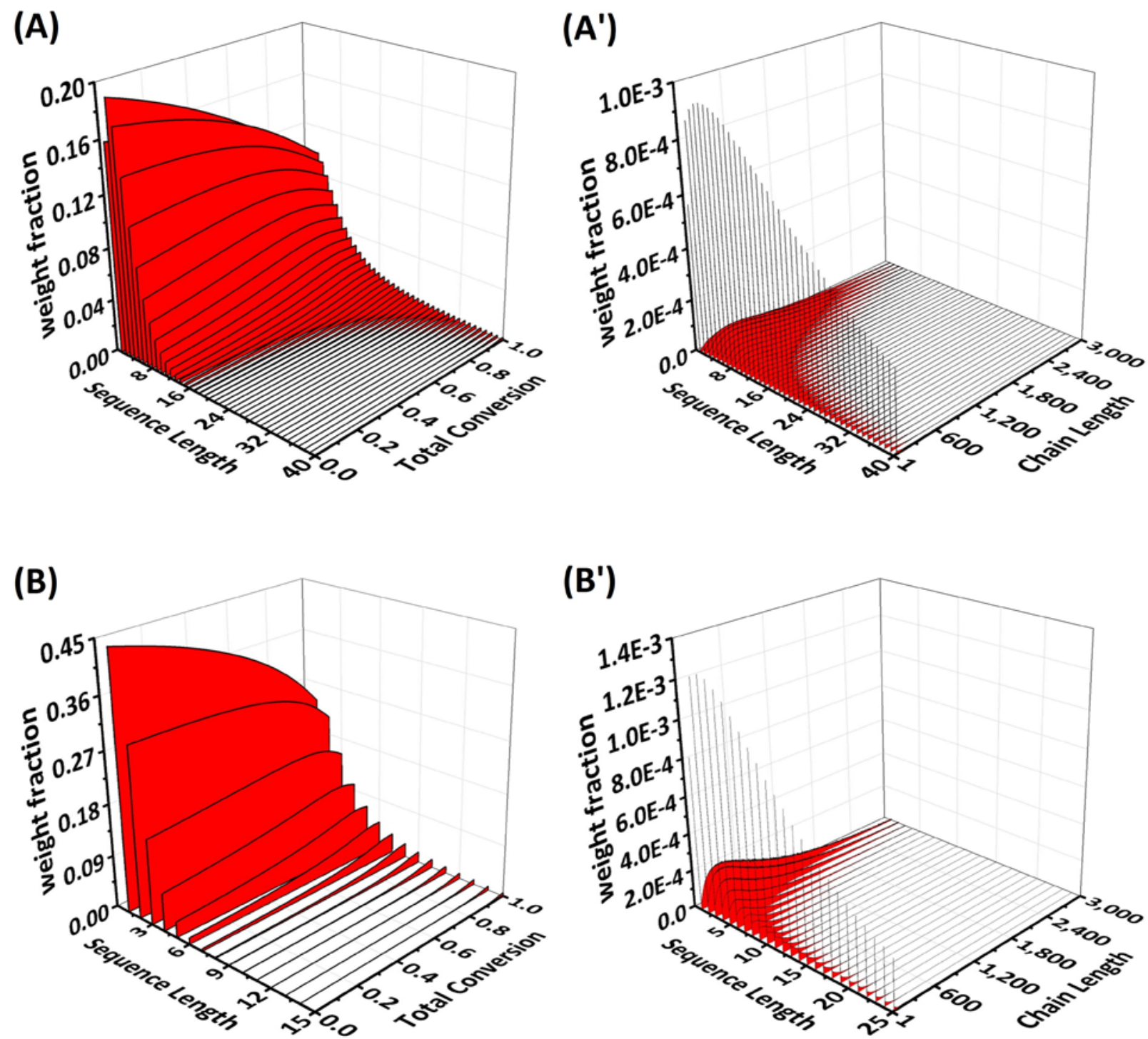

(B')

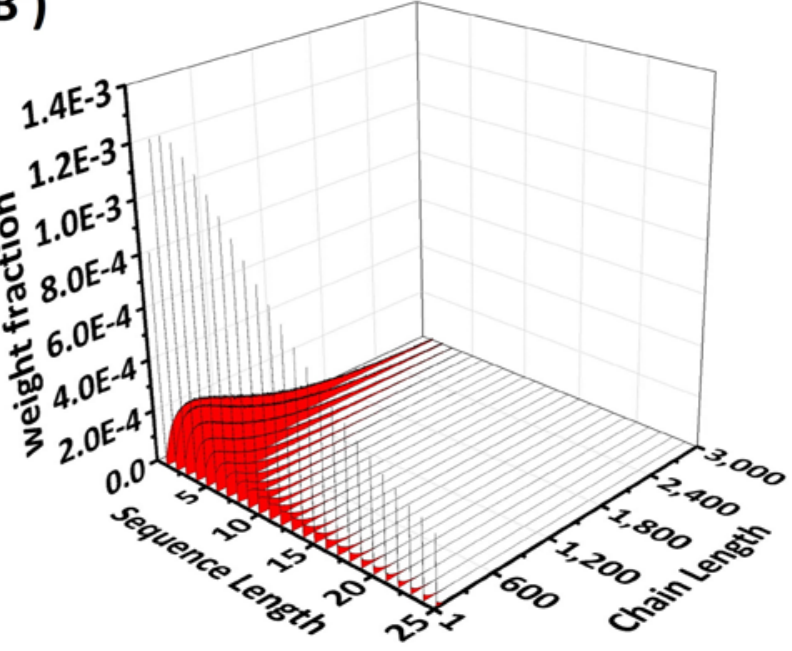

(C)

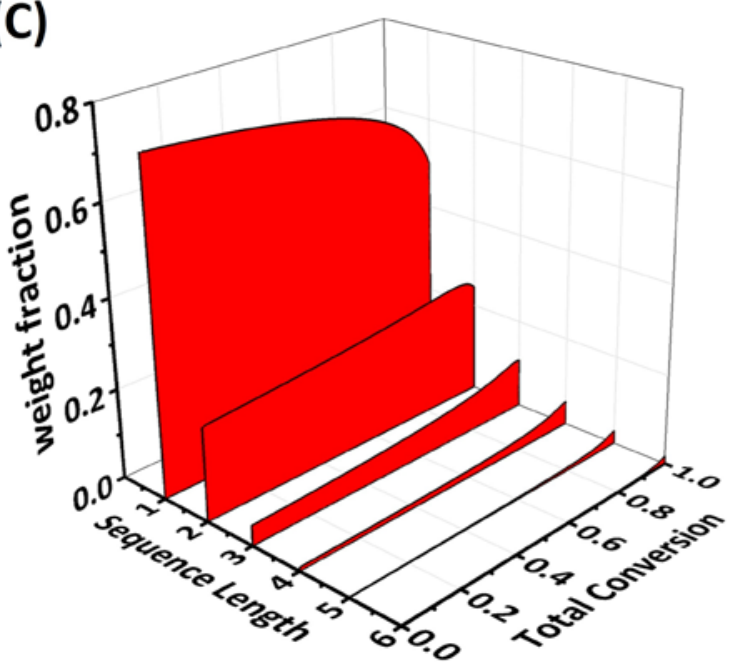

(C')

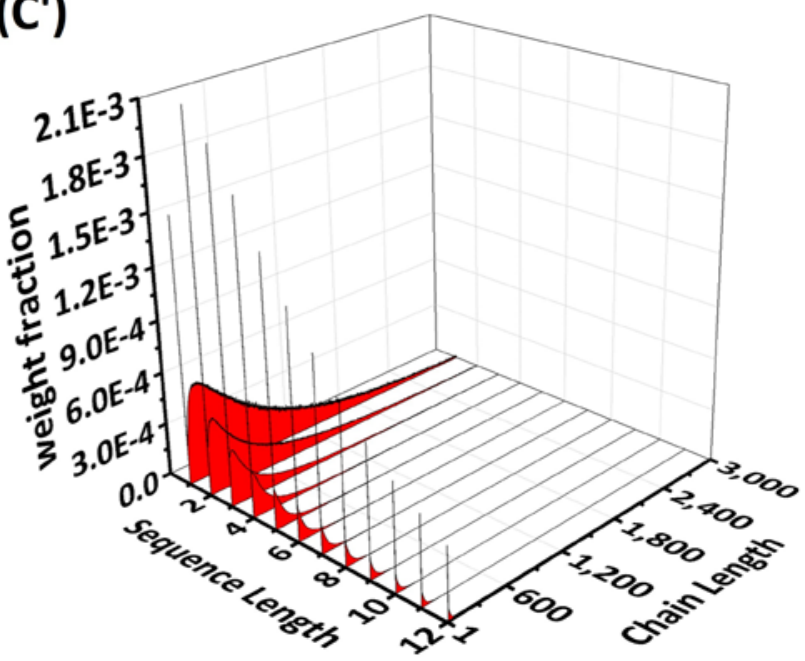


Figure 14. Profiles of evolution of weight fraction of sequence length distribution of $M 2$ under feed composition of 0.25 (A); 0.50 (B); and 0.75 (C) against conversion; and SL-CL weight distribution of $M 2$ for feed composition of $0.25\left(\mathrm{~A}^{\prime}\right) ; 0.50\left(\mathrm{~B}^{\prime}\right)$; and $0.75\left(\mathrm{C}^{\prime}\right) ; r_{1}=2.0$ and $r_{2}=0.5$

\section{REFERENCES}

[1] T. Meyer, J. Keurentjes, "Handbook of Polymer Reaction Engineering", Wiley-VCH, Weinheim, 2005.

[2] A. E. Hamielec, J. F. MacGregor, A. Penlidis, Pergamon Press plc, Comprehensive Polymer Science: the Synthesis, Characterization, Reactions \& Applications of Polymers. 1989, 3, 17.

[3] E. L. Madruga, Progress in Polymer Science 2002, 27, 1879.

[4] Y. Zhao, Y.-W. Luo, B.-G. Li, S. Zhu, Langmuir 2011, 27, 11306.

[5] K. Matyjaszewski, Progress in Polymer Science 2005, 30, 858.

[6] Y. Mohammadi, A. S. Pakdel, M. R. Saeb, K. Boodhoo, Chemical Engineering Journal 2014, 247, 231.

[7] I. Kryven, P. D. ledema, Macromolecular Reaction Engineering 2015, 9, 285.

[8] Y. Mohammadi, M. Najafi, V. Haddadi-Asl, Macromolecular Theory and Simulations 2005, 14, 325.

[9] M. Najafi, V. Haddadi-Asl, Y. Mohammadi, Journal of Applied Polymer Science 2007, 106, 4138.

[10] J. J. Semler, Y. K. Jhon, A. Tonelli, M. Beevers, R. Krishnamoorti, J. Genzer, Advanced Materials 2007, 19, 2877.

[11] J. M. Asua, "Polymer Reaction Engineering", Blackwell Pub., Oxford; Ames, lowa, 2007.

[12] Y. Mohammadi, M. Ahmadi, M. R. Saeb, M. M. Khorasani, P. Yang, F. J. Stadler, Macromolecules 2014, 47, 4778.

[13] M. Ahmadi, M. R. Saeb, Y. Mohammadi, M. M. Khorasani, F. J. Stadler, Industrial \& Engineering Chemistry Research 2015, 54, 8867.

[14] M. M. Khorasani, M. R. Saeb, Y. Mohammadi, M. Ahmadi, Chemical Engineering Science 2014, 111, 211. 
[15] M. R. Saeb, Y. Mohammadi, M. Ahmadi, M. M. Khorasani, F. J. Stadler, Chemical Engineering Journal 2015, 274, 169.

[16] C. Toloza Porras, D. R. D'Hooge, P. H. M. Van Steenberge, M.-F. Reyniers, G. B. Marin, Macromolecular Reaction Engineering 2013, 7, 311.

[17] T. Fujisawa, A. Penlidis, Journal of Macromolecular Science, Pure \& Appl. Chem, Part A 2008, $45,115$.

[18] M. R. Saeb, Y. Mohammadi, A. S. Pakdel, A. Penlidis, Macromolecular Theory and Simulations 2016, 25, 369.

[19] G. G. Odian, "Principles of Polymerization", 2004.

[20] G. Marsaglia, J. Mod. Appl. Stat. Methods 2003, 2, 2.

[21] Y.-N. Zhou, J.-J. Li, Z.-H. Luo, Journal of Polymer Science Part A: Polymer Chemistry 2012, 50, 3052 .

[22] U. Beginn, Polymer 2006, 47, 6880.

[23] A. Keramopoulos, C. Kiparissides, Macromolecules 2002, 35, 4155.

[24] M. M. Wu, "Acrylonitrile Polymers, Survey and Styrene-Acrylonitrile (SAN)", in Kirk-Othmer Encyclopedia of Chemical Technology, John Wiley \& Sons, Inc., 2000. 